\title{
Development and optimization of Lysis gene E as a counter-selection marker with high selection stringency
}

\author{
Wei Chen ${ }^{1}$, Ruyi Chen ${ }^{1}$, Ling $\mathrm{He}^{1}$, and Xiaotong $\mathrm{Wu}^{1}$ \\ ${ }^{1}$ Guangdong Pharmaceutical University
}

September 24, 2021

\begin{abstract}
Seamless modification of bacteria chromosome is widely performed both in theoretical and in practical research, for this purpose, excellent counter-selection marker genes with high selection stringency are needed. Lysis gene E from bacteriophage PhiX174 was developed and optimized as a counter-selection marker in this paper. Lysis gene $\mathrm{E}$ was firstly constructed under the control of pL promoter. At $42{ }^{\circ} \mathrm{C}$, Lysis gene E could effectively kill Escherichia coli. Seamless modification using E as a counter-selection marker also successfully conducted. It also works in another Gram-negative strain Serratia marcescens under the control of Arac/PBAD regulatory system. Through combining lysis gene E and kil, the selection stringency frequency of pL-kil-sd-E cassette in E. coli arrived at $4.9 \times 10-8$ and $3.2 \times 10-8$ at two test loci, which is very close to the best counter-selection system, inducible toxins system. Under the control of Arac/PBAD, selection stringency of PBAD-kil-sd-E in S. marcescens arrived at the level of 10-7 at four test loci. By introducing araC gene harboring plasmid pKDsg-ack, 5- to 18- fold improvement of selection stringency was observed at these loci, and a surprising low selection stringency frequency 4.9x10-9 was obtained at marR-1 locus, the lowest selection stringency frequency for counter-selection reported so far. Similarly, at araB locus of E. coli selection stringency frequency of PBAD-kil-sd-E was improved to 3x10-9 after introducing plasmid pKDsg-ack. In conclusion, we have developed and optimized a newly universal counter-selection marker based on lysis gene E. The best selection stringency of this new marker exceeds the inducible toxins system several fold.
\end{abstract}

Development and optimization of Lysis gene $E$ as a counter-selection marker with high selection stringency Running title: High stringent counter-selection marker based on lysis gene $E$

Wei Chen ${ }^{1,2^{*}}$, Ruyi Chen ${ }^{1}$, Ling $\mathbf{H e}^{2}$, Xiaotong $\mathbf{W u}{ }^{1}$

1, Guangdong Province Key Laboratory for Biotechnology Drug Candidates, School of Biosciences and Biopharmaceutics, Guangdong Pharmaceutical University, Guangzhou 510006, China

2, Guangdong Key Laboratory of Pharmaceutical Bioactive Substances, Guangdong Pharmaceutical University, Guangzhou 510006, China

${ }^{*}$ Corresponding author: Wei Chen, $\mathrm{PhD}$

School of Biosciences and Biopharmaceutics, Guangdong Pharmaceutical University

Guangzhou 510006, China

Tel.: +86 2039352201

E-mail : thinker98@163.com

\section{Abstract}


Seamless modification of bacteria chromosome is widely performed both in theoretical and in practical research, for this purpose excellent counter-selection marker genes with high selection stringency are needed. Lysis gene $E$ from bacteriophage PhiX174 was developed and optimized as a generic counter-selection marker in this paper.

Lysis gene $E$ was firstly constructed under the control of $p L$ promoter. At $42{ }^{\circ} \mathrm{C}$, it could effectively killEscherichia coli. Seamless modification using $E$ as a counter-selection marker was successfully conducted. It also works in another Gram-negative strain Serratia marcescens under the control of Arac/ $\mathrm{P}_{\mathrm{BAD}}$ regulatory system. Through combining lysis gene $E$ and $k i l$, the selection stringency frequency of $p L-k i l-$ $s d-E$ cassette in $E$. coliarrived at $4.9 \times 10^{-8}$ and $3.2 \times 10^{-8}$ at two test loci, which is very close to the best counter-selection system, inducible toxins system. Under the control of Arac $/ \mathrm{P}_{\mathrm{BAD}}$, selection stringency of $P_{B A D}$-kil-sd-E in S. marcescens arrived at the level of $10^{-7}$ at four test loci. By introducing araC gene harboring plasmid pKDsg-ack, 5- to 18- fold improvement of selection stringency was observed at these loci, and a surprising low selection stringency frequency $4.9 \times 10^{-9}$ was obtained at mar $R-1$ locus, the lowest selection stringency frequency for counter-selection reported so far. Similarly, at araB locus of $E$. coli selection stringency frequency of $P_{B A D}-k i l-s d-E$ was improved to $3 \times 10^{-9}$ after introducing plasmid pKDsg-ack.

In conclusion, we have developed and optimized a newly universal counter-selection marker based on lysis gene $E$. The best selection stringency of this new marker exceeds the inducible toxins system several fold.

Keyword: Counter-selection, Selection stringency frequency, lysis gene $E$, Red recombination, metabolic engineering

\section{Background}

Foreigner DNA fragments are usually needed to be introduced into bacteria. They can be constructed into replicative plasmids, plasmids are inherently unstable, limiting the application of this strategy in researches with high requirements for the stability of results. As a better alternative, exogenous DNA fragments should be irreversibly incorporated into chromosome or other stable DNA molecule inside the cell (Heap et al., 2012). For this sake, DNA fragments of interest could be combined with positive selection markers, typically using an antibiotic resistance gene, allowing desired recombinant cells to be selected and isolated after a single-crossover or double-crossover recombination. Positive selection markers can also be removed through specific procedure, such as FLP- or CRE-mediated recombination (Meyers, Lewandoski, \& Martin, 1998), but frt or loxp site will leave behind inevitably (Lee et al., 2001). Generally speaking, for the sake of biosecurity or eliminating unanticipated effects, residual of resistance genes or frt and loxp scars on the genome is not expected during functional genome analysis and metabolic engineering research.

This issue can be overcome using a two-step selection method by combining positive-negative selection cassette. In particular, a positive-negative selection cassette could be introduced and recombinants are selected according to positive selection markers, then exogenous DNA fragments are transformed into cells to replace the above-mentioned cassette and the final desired recombinants are selected against the counterselection gene.

Many positive selection markers are highly efficient so recombinants could be easily acquired in the first recombination event. In contrast, counter-selection markers are not so satisfactory. The main problem of counter-selection markers is that high background causes great difficulties for counter-selection, especially in the case of low recombination efficiency (Imam et al., 2000; Muyrers et al., 2000; Y. Zhang, F. Buchholz, J. P. Muyrers, \& A. F. Stewart, 1998). Several research groups are being dedicated to developing and optimizing better counter-selection markers(DeVito, 2008; Ma et al., 2015; Wang et al., 2014; Wong et al., 2005) and some novel counter-selection marker genes have been reported. Among them tetA -sacB and inducible toxins system are the most outstanding (Khetrapal et al., 2015; Li, Thomason, Sawitzke, \& Costantino, 2013). It is worth mentioning that inducible toxins system possesses the highest selection stringency $\left(1.1 \times 10^{-8}\right.$ to $\left.3.31 \times 10^{-8}\right)$ which even comes up to that of positive antibiotic markers in some strains (Khetrapal et al., 2015). Despite these achievements, excellent counter-selection markers are still scanty. In view of the situation, we are motivated to develop robust counter-selection marker and provide new strategy 
for optimizing counter-selection efficiency.

Lysis gene $E$ of bacteriophage PhiX174 was discovered to have a role in lysing Escherichia coli in 1966 for the first time (Hutchison \& Sinsheimer, 1966), and the subsequent researches shows that expression of this gene is sufficient to cause lysis of E. coli (Henrich, Lubitz, \& Plapp, 1982; Young \& Young, 1982). Lysis gene $E$ codes for a 91-aa membrane protein with hydrophobic moieties at its $\mathrm{N}$-terminal end that oligomerizes into a transmembrane tunnel structure(Blasi, Linke, \& Lubitz, 1989; Witte \& Lubitz, 1989). This specific tunnel structure is associated with the fusion of the inner and outer membranes of Gram-negative bacteria, leaving behind empty envelopes devoid of all cytoplastic content (Hajam, Dar, Won, \& Lee, 2017; Witte, Wanner, Sulzner, \& Lubitz, 1992). Non-living Gram-negative bacterial empty envelopes caused by E protein are knows as Bacterial Ghosts (BG), representing a potential platform both for potent candidate vaccines and for technical applications in white biotechnology (Hajam et al., 2017; Langemann et al., 2010; Won, Hajam, \& Lee, 2017). BGs of many Gram-negative strains have been prepared successfully, including Salmonella typhimurium, Salmonella enteritidis ,Bordetella bronchiseptica, Vibrio cholerde ,Mannbeimia haemolytica , Pseudomonas aeruginosa, etc (Langemann et al., 2010). In view of the universal and potent killing effect mediated by lysis protein $\mathrm{E}$ in Gram-negative strains, we focus our effort on developing lysis $E$ gene as a universal marker gene to try to expand the existing counter-election tool chest, which is especially useful for host strains lack of facile genetic tools.

This study reports on the introduction of lysis gene $E$ of PhiX174 to create "seamless" genetic manipulations in Gram-negative strains. Under the control of the lambda $p L$ promoter and $\mathrm{AraC} / \mathrm{P}_{\mathrm{BAD}}$ inducible system, lysis gene $E$ can effectively promote the death of $E$. coli and Serratia marcescens, and the selection stringency of lysis gene $E$ is similar to the next best reported tetA-sacB double-negative selection system. By coexpressing lysis gene $E$ and kilnegative gene (kil-sd-E counter-selection cassette) through a classic RBS (Ribosome Binding Site) sequence, the selection stringency frequency is even comparable with the best counter-selection system, inducible toxins system. By introducing $a r a C$ gene harboring plasmid, the selection stringency frequency of kil-sd-E arrives at the level of $10^{-9}$ both in E. coli and in S. marcescens . This is the lowest selection stringency frequency for counter-selection reported so far, exceeding the best counter selection system, inducible toxins system, several fold.

Due to the universal lethal of expression of lysis protein E, counter selection cassette based on lysis $E$ or $k i l$ $s d-E$ could be applicable to many, if not all, Gram-negative strains. And the strategy reported in this article of combining two short counter-selection genes could be used to increase the counter-selection efficiency of other negative markers, which can provide more powerful tools to the genome editing toolbox.

\section{Methods}

\section{Bacteria strains and plasmids "}

All bacteria strains used in this paper are listed in Table 1. E. coli Strains MG1655 are generous gifts from Professor Sheng Yang.E. coli Strains harboring kil counter-selection system was constructed previously in our lab. S. marcescens strain GY1 was isolated previously in our lab. Plasmids pKD46 and pSim6 are generous gifts from Doctor Donald L. Court. Plasmid pKDsg-ack was purchased from Addgene (www.addgene.org, 62654).

\section{Medium and antibiotics}

Standard cultures were propagated in Luria Bertani (LB) broth or LB agar with or without antibiotics. Antibiotics are added at the following concentrations: $50 \mu \mathrm{g} / \mathrm{mL}$ of ampicillin, $25 \mu \mathrm{g} / \mathrm{mL}$ of kanamycin and $50 \mu \mathrm{g} / \mathrm{mL}$ of gentamicin for E. coli ; $1 \mathrm{mg} / \mathrm{mL}$ of ampicillin, $100 \mu \mathrm{g} / \mathrm{mL}$ of kanamycin and $50 \mu \mathrm{g} / \mathrm{mL}$ of gentamicin for $S$. marcescens .

\section{Primer designation}

All oligos were synthesized in Sangon Biotech (China) and listed in Table 2. 
High-fidelity polymerase PrimeSTAR ${ }^{\circledR}$ GXL DNA polymerase (Takara, Japan) were used for amplifying linear dsDNA targeting fragments. Primers used to produce these targeting fragments were chimeric primers containing two parts: 38 bases homologous to the sequence surrounding the target locus were designed at 5 ' end of primers, while about 20 bases of amplifying sequence were at 3' end. After purification following PCR amplification, linear dsDNA was electroporated into cells for Red recombination. Single-stranded DNA, sspigA-F, used to delete $P_{B A D}-E-G m^{R}$ or $P_{B A D}$-kil-sd-E-Gm ${ }^{R}$ selection/counter-selection cassettes inserted into the pigAlocus of $S$. marcescens was reported in our previous study (W. Chen, Chen, \& Cao, 2021).

\section{Preparation of electroporation competent and cell transformation}

$600 \mu \mathrm{l}$ of overnight cultures were 1:50 diluted into $30 \mathrm{ml}$ fresh $\mathrm{LB}$ medium and grown at $30{ }^{\circ} \mathrm{C}$ to an $\mathrm{OD}_{600}=0.4-0.6$. If Red recombinases harbored by plasmid pSim6 were needed to be expressed, cultures were then shaken at $42{ }^{\circ} \mathrm{C}$ for $15 \mathrm{~min}$ in a water bath. Cultures were centrifugated at $4{ }^{\circ} \mathrm{C}$ for 15 min, washed $3^{\sim} 4$ times with $20 \mathrm{ml}$ of cold deionized water. Cell pellet was ultimately suspended in $0.5 \mathrm{ml}$ of deionized water. $40 \mu \mathrm{l}$ of fresh prepared competent cells was used for an electroporation reaction. Electroporation was carried out using Gene-Pulser (Bio-Rad Laboratories, USA) at $1.8 \mathrm{kv}, 25 \mu \mathrm{F}$ and $200 \Omega$. Electroporated cells were cultured in $2 \mathrm{ml}$ of LB broth with shaking for $2 \mathrm{~h}$ and then spread onto corresponding counter-selective agar plates. In detail, for counter-selection marker controlled by $\mathrm{AraC} / \mathrm{P}_{\mathrm{BAD}}$ inducible system, cells were spread onto LB agar plates supplemented with $0.4 \%$ arabinose and grown at $30{ }^{\circ} \mathrm{C}$; for counter-selection marker controlled by pL promoter, cells were spread onto normal LB agar plates at cultivated at $42{ }^{\circ} \mathrm{C}$.

\section{Verification of recombinants after positive or negative selection}

After spread onto corresponding selective agar plates, colony PCR was used for identification of positive recombinants. A general principle for designing diagnostic PCR primer is: one primer is surrounding the target region and the other is inside the donor DNA. Two paired primers surrounding each side of the target region respectively were also designed in some case to display the different size of target DNA between the original strains and positive recombinants.

\section{Assessment of the lethal effect}

Streak cultivation was performed to analyze lethal effect of counter-selection marker under the control of $p L$ promoter: colony grown well at $30{ }^{\circ} \mathrm{C}$ were streak-cultured onto

plate and placed at $42{ }^{\circ} \mathrm{C}$ to observe the lethal effect, and another plate with the same treatment was placed at $30{ }^{\circ} \mathrm{C}$ as a control.

To assess the lethal effect of counter-selection marker under the control of AraC $/ \mathrm{P}_{\mathrm{BAD}}$ inducible system, colony was inoculated into $2 \mathrm{ml}$ of LB broth supplemented with or without $0.4 \%$ arabinose and incubated for 12 to $16 \mathrm{~h}$.

Spot titers experiment was performed according to previous report (Haeusser et al., 2014). In short, overnight culture was serially 10 -fold diluted and $10 \mu \mathrm{L}$ of diluted culture as indicated in the figures was spotted on the plates under corresponding counter-selection conditions, and another plate without the stress of counterselection was used as a control.

\section{Selection stringency analysis}

Selection stringency measurement is according to the report of Khetrapal with some minor modifications (Khetrapal et al., 2015). In brief, overnight cultures were 1:50 diluted and grown at $30^{\circ} \mathrm{C}$ to $\mathrm{OD}_{600}=0.4^{\sim} 0.6$. $10^{4}-10^{5}$ diluted cultures were spread onto non-restrictive LB agar plates and grown at $30^{\circ} \mathrm{C}$ to quantify the number of surviving bacterial (CFU) in the overnight cultures. At the same time, about $10^{10}$ re-suspended cells after centrifugation were spread onto appropriate restrictive plates or placed at specific counter-selection conditions to quantify the number of colonies which escape the lethal effect of counter-selection gene.

Selection stringency frequency was determined by dividing the number of escaping colonies on restrictive plates by the CFU that grew on non-restrictive conditions. 


\section{Plasmid curing and electroporation}

For the curing of plasmid pSim6, cells carrying pSim6 was inoculated into $2 \mathrm{ml}$ of LB without ampicillin addition and incubated for 12 to $16 \mathrm{~h}$ at $37^{\circ} \mathrm{C}$. Cells equivalent in quantity to $10^{-4} \mu \mathrm{L}$ of cultures were spread onto LB plates and grown at $37{ }^{\circ} \mathrm{C}$. The colonies were confirmed as cured by determining their sensitivity to ampicillin ( $1 \mathrm{mg} / \mathrm{mL}$ for $S$. marcescens and $100 \mu \mathrm{g} / \mathrm{mL}$ for E. coli ) and by PCR identification of the elimination of pSim6.

Plasmid pKDsg-ack was then electroporated into the above pSim6-eliminated cells and spread onto LB agar plates supplemented with ampicillin ( $1 \mathrm{mg} / \mathrm{mL}$ for $S$. marcescens and $100 \mu \mathrm{g} / \mathrm{mL}$ for E. coli ).

\section{Results:}

\section{Potential of lysis gene $\mathrm{E}$ as a counter-selection marker gene}

Several previous studies have reported that expression of lysis gene $E$ could promote the death of many Gram-negative bacteria through fusion of the inner and outer membranes. These results show that the product of lysis gene $E$ has a universal killing effect on Gram-negative bacteria, which prompt us to explore the potential of lysis gene $E$ being a generic counter-selection marker gene for genetic modifications in Gramnegative bacteria. For this purpose, expression of lysis gene $E$ should be controllable. In our previous work, kil counter-selection cassette under the control of promoter $p L$ function well in $E$. coli (Wei Chen et al., 2019). We want to test the killing effect of lysis gene $E$ under the same condition. Therefore, plasmid pBBR1-MC5 and the CDS (Coding Sequence) of lysis gene $E$ were firstly ligated after digestion with Sph I and Nco I. Thus, the CDS of lysis gene $E$ is linked with the gentamycin resistance gene $G m^{R}$ in the newly constructed plasmid pBBR1-E (Fig. 1A). Using dsDNA mediated Red homologous recombination, PCR amplified $E-G m^{R}$ (including gentamycin resistance gene and CDS of lysis gene E) cassette was then used to substitute the kil CDS of tet-kil double selection cassette of $E$. coli MG-10 (Figure 1B). The goal strain, named as CWE-1, was successfully constructed (Fig 1C). To exclude the interference of $G m^{R}$, tet-pl-E cassette was amplified and used to substituted the CDS of ack. The goal strain was names as CWE-2 (Fig. 1D).

According to our expectation, since expression of lysis gene $E$ in CWE-2 is driven by the $p L$ promoter, which is in turn under the control of temperature-sensitive cI857 repressor (Wei Chen et al., 2019; Yu et al., 2000), lysis gene $E$ could be induced because of the loss-of-function of the repressor cI857 at $42{ }^{\circ} \mathrm{C}$. If expression of lysis gene $E 42{ }^{\circ} \mathrm{C}$ could effectively cause the death of host cell, it has the potential of being a counter-selection marker. As shown in Fig. 2A, after streak culture no growth traces of CWE-2 can be found at LB agar plate placed at $42{ }^{\circ} \mathrm{C}$. This is similar to the positive control strain MG-10, in which counterselection marker gene kil constructed was induced to kill host strain (Wei Chen et al., 2019). At the same time, obvious growth of the negative control strain MG1655 [pSim6] appeared on agar plate. In contrast, all strains grew well at $30{ }^{\circ} \mathrm{C}$. It indicates that our newly constructed lysis gene $E$ expression cassette meet the requirement of a negative-selection marker gene: having no negative influence in the growth of host cell under non-induced conditions, while effectively killing its host under induced conditions. This is also verified by spot titers experiment (Fig. 2B). Our results indicate that lysis gene $E$ has good potential to be a counter-selection marker.

\section{Seamless modification testing of thelysis gene E counter-selection cassette in E.coli}

To directly investigate the effect of lysis gene $E$ in counter-selection, PCR amplified 1,000 bp DNA fragment of catenin gene from Helicoverpa armigera with $38 \mathrm{bp}$ of short homologous arms was used to substitute the foreign tet-pL-E at the ack locus of CWE-2. At $42{ }^{\circ} \mathrm{C}$ sparsely spread colonies were shown in the control group which was electroporated without adding DNA, whereas much more colonies appeared in the group electroporated with dsDNA (Fig. 2C). The following colony PCR showed that 9 out of 10 randomly selected colonies were all correct recombinants (Fig. 2D). These results suggest that seamless modification using lysis gene $E$ as a counter-selection marker is feasible in $E$. coli .

\section{Application of}




\section{lysis gene E counter-selection cassette in $S$. marcescens}

A counter-selection marker would be more attractive if it has a universal application. In the interest of generality, application of lysis gene $E$ counter-selection cassette in other bacteria should be tested.

S. marcescens, a rod-shaped gram-negative bacterium, have the ability to produce a variety of valuable metabolites, such as prodigiosin, chitinase and serratiopeptidase(Emruzi et al., 2018; Pan et al., 2019; VelezGomez, Melchor-Moncada, Veloza, \& Sepulveda-Arias, 2019; Yip et al., 2019). We select S. marcescens to test the counter-selection effect of lysis gene $E$ cassette. Unfortunately, high-temperature condition like 42 ${ }^{\circ} \mathrm{C}$ is not suitable for the growth ofS. marcescens (W. Chen et al., 2021), We resorted to the AraC/P $\mathrm{BAD}$ regulatory system which function well in this species. At first, $E-G m^{R}$ fragment with short homologous arms was amplified and transformed into competent MG1655[pKD46] to substitute the CDS of Red recombinases in plasmid pKD46 to construct the goal plasmid pKD-EG. Therefore, expression of lysis gene $E$ is thoroughly controlled by AraC/PBAD inducible system (Fig. 3A). After identification (Fig. 3B), plasmid pKD-EG was transformed into $S$. marcescens GY1 for functional testing. GY1[pKD-EG] grew well in LB broth, but it cannot survive in LB broth supplemented with $0.4 \%$ arabinose, and the LB broth is very clear even $18 \mathrm{~h}$ after inoculation (Fig. 3C). This result indicates that expression of lysis gene Ethrough AraC/ $\mathrm{P}_{\mathrm{BAD}}$ regulatory system could effectively kill its host.

In genomic seamless modification, counter-selection marker usually works in single copy. Therefore, the counter-selection effect of lysis gene $E$ inserted in the chromosome must be evaluated. The $2,303 \mathrm{bp} P_{B A D}-E-$ $G m^{R}$ (including AraC/PBAD regulatory system, CDS of lysis gene $\mathrm{E}$ and gentamycin resistance gene $G m^{R}$ ) double selection cassette was therefore inserted into pigA CDS of $S$. marcescens. The successfully constructed strain was named as GY4 (Fig. 3D). Obvious differences can be seen when GY4 grown in LB broth without or with the addition of $0.4 \%$ arabinose (Fig. 3E). This result indicates that single copy of counter-selection marker gene $E$ is also efficient. It is further verified by the spot dilution experiment: GY4 grew well on LB plate, while it cannot survive on arabinose added plate and only a faintly layer of dead cells was observed (Fig. 3F). It should be noted thatpigA is an indispensably gene for the synthesis of prodigiosin, a kind of red pigment, therefor GY1 shows red phenotype while pigAgene mutated GY4 is white.

Having verified the lethality of chromosomal expressing lysis gene $E$ in $S$. marcescens, reverse mutation experiment was finally conducted to test its counter-selection effect in genomic modification. Using ssDNA mediated Red recombination, we tried to repair the insertion inactivation of pigA gene in GY4 (Fig. S1). Numerous red colonies dotted with a few white colonies were observed after transported GY4 with ssDNA, while only sparse white colonies appear in the control group without adding ssDNA (Fig. 4A). Colony in red indicates that the ability of synthesizing prodigiosin was repaired due to successful reverse mutation, which is verified by colony PCR identification (Fig. 4B).

dsDNA mediated homologous recombination was also tested. Partial fragment of T7 RNA polymerase $\left(^{\sim}\right.$ $1,000 \mathrm{bp}$ in length) was amplified and transported into GY4 to substitute the $P_{B A D}-E-G m^{R}$ cassette. 4 out of 10 of randomly selected colonies were true recombinants (Fig. 4C).

All these results suggest that lysis gene $E$ counter-selection cassette can be used in $S$. marcescens for genomic seamless modification.

\section{Improving selection stringency frequency through combining $E$ and kil}

In genomic seamless modification, selection stringency frequency is a decisive factor that influences counterselection and therefor an indicator for evaluating an excellent counter-selection marker(Khetrapal et al., 2015). The higher the selection stringency frequency is, the lower the background it causes. Low background is very conducive to the selection of recombinants during seamless modification. The selection stringency frequency of lysis $E$ under the control of promoter $p L$ at ack locus in MG1655 is about $2.7 \times 10^{-7}$ (Fig. $5 \mathrm{~A}$ ), performing better than thekil counter-selection cassette constructed in our previous work (Wei Chen et al., 2019). However, it is still much lower than the best counter-selection system, inducible toxins system(Khetrapal et al., 2015). 
We tried to increase the selection stringency by combing the two counter-selection marker genes. At first, CDS of lysis gene $E$ with a consensus RBS sequence (AAGGAGATATACAT) and gentamycin resistance gene $G m^{R}$ were inserted immediately behind the stop codon of kil gene at ack locus of E. coliMG-10. In this constructed strain CWE-3, kil and $E$ were expressed as a bi-cistron under the control of promoter $\mathrm{pL}$ and repressor cI857 (Fig. S2). We named this combining counter-selection cassette askil-sd-E. Then the selection stringency of these counter-selection systems was compared. At the ack locus, selection stringency frequency of $E$ is $2.7 \times 10^{-7}$, several fold lower than that of $k i l\left(8.7 \times 10^{-7}\right)$. While selection stringency frequency ofkil-sd-E was significantly decreased to $4.9 \times 10^{-8}$ (Fig. 5A), which is very close to the best reported inducible toxins system.

In consideration that insertion sites may influence stringency, another non-essential gene locus araB were selected for the further analysis. Selection stringency frequency of $E\left(2.9 \times 10^{-6}\right)$ and $k i l\left(2.1 \times 10^{-6}\right)$ are almost at the same level. But selection stringency frequency of kil-sd-E dropped sharply to $3.2 \times 10^{-8}$ at this locus, about 65- to 90- fold lower than the above two counter-selection system (Fig. 5B). This result hinds that co-expression of $k i l$ and $E$ in the form of bi-cistron can significantly increase their selection stringency in $E$. coli to the degree to the best reported inducible toxins system.

To explore whether it also works in other bacteria, S. marcescenswas used for the subsequent research. At first, $E-G m^{R}$ and $k i l-s d-E-G m^{R}$ were amplified from plasmid pBBR-E and strain CWE-3 separately and then were used to substitute the CDS of $a r a B$ to construct the goal strain MG-4A and MG-4B (Fig. S3). Sole expression of $E$ or co-expression of kil and $E$ were both under the control of $\mathrm{AraC} / \mathrm{P}_{\mathrm{BAD}}$ in the two strains. After adding $0.4 \%$ arabinose into LB broth, both MG-4A and MG-4B can't grow normally. In particular, the LB broth was very clear in the MG-4B cultivating tube $18 \mathrm{~h}$ after inoculation, while slightly turbid cultivated bacteria can be seen in the MG-4A cultivating tube (Fig. 5C). These results indicate that $E$ and kil-sd-E counter-selection marker under the control of AraC/P $\mathrm{P}_{\mathrm{BAD}}$ function well in $E$. coli . It also hints that co-expression of $k i l$ and $E$ is of better lethal effect than the sole expression of $E$.

$\mathrm{AraC} / \mathrm{P}_{\mathrm{BAD}}-k i l-s d-E-G m^{R}$ cassette was then introduced into $S$. marcescens to insert into the CDS ofpigA . The goal strain was named as GY5. Selection stringency frequency of GY5 $\left(1.4 \times 10^{-7}\right)$ is about 10 - fold lower than that of GY4 $\left(1.9 \times 10^{-6}\right)$ (Fig. 5D). The result hints that counter-selection system in GY5 should perform better than GY4. This was then verified by the ssDNA mediated mutation repair: compared with GY4 (Fig. 4A), no matter in the control group or in the ssDNA added group, fewer white colonies were shown on GY5 (Fig. 5E). When 1,000 bp T7 RNA polymerase gene fragment was used to substitute the AraC/ $\mathrm{P}_{\mathrm{BAD}}$-kil-sd-E-Gm ${ }^{R}$ in GY5, 6 out of 10 randomly selected colonies were correct (Fig. 5G). The ratio of correct recombinants is also higher than that in GY4 (Fig. 4C).

All these results show that co-expression of kil and $E$ can elevate the selection stringency by orders of magnitude in multiple species and decrease the number of escaping colonies during seamless modification.

\section{Improving selection stringency frequency of $k i l-s d-E$ by introducing plasmid pKDsg-ack}

Selection stringency frequency of kil-sd-E under the control of $\mathrm{AraC} / \mathrm{P}_{\mathrm{BAD}}$ system was at the level of $10^{-7}$ at the pigA locus (Fig. 5H). We analyzed other three loci of $S$. marcescens (The first gene code for MarR family transcriptional regulator, we named it as $\operatorname{mar} R-1$; the second gene $\operatorname{rcs} A$ codes for DNA-binding transcriptional activator; the last gene code for a hypothetical protein, we named it as hyp-1), the results are similar (Fig. 6A), other than a very low stringency frequency occurred atmarR-1 locus $\left(2.5 \times 10^{-8}\right)$. Although selection stringency frequency of $10^{-7}$ is relatively high and even better than the next best tetA-sac $B$ system in $E$. coli , it is still not as good as kil-sd-E counter-selection marker controlled by $p L$ promoter.

To provide a more powerful generic counter-selection marker, wo want to elevate the selection stringency of this system. Promoter $P_{B A D}$ is dual regulated by transcription factors Arac. Arac can bind to $P_{B A D}$ and act as repressor or inducer depending on the absence or presence of arabinose. The increase in the number of transcription factor AraC can not only promote the expression of gene it controlled after addition of arabinose, but also reduce leakage expression of gene it controlled in de absence of inducer. Therefore, we suspected that the existence of multiple ara $C$ genes is very likely to be beneficial to the enhancement of 
selection stringency.

Plasmid pKDsg-ack which carried the araC gene (Reisch \& Prather, 2015) was introduced into the host cell after the original plasmid pSim6 being cured under 37degC cultivation. In MG1655, selection stringency frequency of kil-sd-E at $a r a B$ locus in pKDsg-ack harboring strain decreased surprisingly to $3 \times 10^{-9}$, This is the lowest selection stringency frequency reported so far. And it is about 170- fold lower than that of pSim6 harboring strain (Fig. 6B). It must be pointed out that plasmid pKDsg-ack is not lethal to host in the presence of arabinose (Fig. S4).

In S. marcescens, selection stringency frequency of $k i l-s d-E$ reached $10^{-8}$ to $10^{-9}$ at all these loci, about $10-$ fold lower than before. In detail, the selection stringency frequency at the $p i g A, r c s A$, andhyp loci was $3.1 \times 10^{-8}, 4.7 \times 10^{-8}$ and $2.5 \times 10^{-8}$ (Fig. $6 \mathrm{C}$ ). A surprisingly low selection stringency frequency, $4.8 \times 10^{-9}$ (Fig. $6 \mathrm{C})$, occurred at the mar locus.

\section{Discussion}

Classic genomic modification to knockout out genes is usually conducted by inserting antibiotic resistance markers. This is simple and easy to implement. However, the existence of unwanted antibiotic resistance marker genes may leave some unexpected effects, which is not expected by researchers in metabolic engineering and functional genome analysis. Through two-step selection/counter-selection method, target genes can be edited without alerting other parts of the chromosome. This unmarked mutation, known as "seamless modification", overcomes the above-mentioned shortcomings.

Although many counter-selection markers have been developed, such assacB, ccdb, I-SceI , galk, mazF (Z. Chen, Ling, 83 Shang, 2016; DeVito, 2008; Muyrers et al., 2000; Van Zyl, Dicks, 83 Deane, 2019; Wang et al., 2014; Warming, Costantino, Court, Jenkins, 83 Copeland, 2005) etc., excellent markers are still limited. Counter-selection genes suffer from some problems which limit their application in genomic modification, among which high background is a serious obstacle. For example, although sacB is most widely used, researchers have to face low positive rates due to the spontaneous mutations that inactivate sacB (Khetrapal et al., 2015; Y. Zhang, F. Buchholz, J. P. Muyrers, \& A. F. J. N. g. Stewart, 1998). Therefore, low background is an important consideration when counter-selection genes are to be choose.

Selection stringency frequency is an indicator that shows the level of background during counter-selection. It has a crucial role of determining the ratio of correct recombinants in counter-selection (Wei Chen et al., 2019). Selection stringency is reflecting by calculating the fraction of viable colonies that escape the selection stress. The smaller the number is, the higher the stringency is (Khetrapal et al., 2015). So far, inducible toxins system performs best among all the developed counter-selection system, followed by tetA-sacBcassette. As for toxins system, the best selection stringency frequency achieved in $E$. coli is $1.11 \times 10^{-8}$, which is exceeds the next best reported tet $A-s a c B$ system up to 60- fold. The selection stringency frequency even approaches that of kanamycin resistance gene $\left(2.4 \times 10^{-9}\right)$ (Khetrapal et al., 2015). Excellent counter-selection markers like inducible toxins system will greatly improve the positive rates and avoid wasting time on redundant work during genomic seamless modification.

Inspired by the inducible toxins system, in this paper we developed a powerful generic counter-selection system for genomic modification, especially for some species which lack efficient genetic tools. Lysis gene $E$ is of general killing effect on Gram-negative bacteria. Given the speculation that counter-selection based on a universal lethal gene would be applicable generally. We focused our attention on the developing and optimizing of lysis $E$ as a counter-selection marker. Expression of lysis gene $E$ under the control of $p L$ promoter efficiently killed its host. dsDNA mediated seamless modification were also successfully performed to substitute target region, and the ratio of correct recombinants is higher than kilcounterselection marker constructed previously in our lab (Wei Chen et al., 2019). In S. marcescens, lysis gene $E$ also can be used for counter-selection marker both through ssDNA and dsDNA mediated recombination. However, the ratio of correct recombinants through dsDNA mediated recombination is lower than that in $E$. coli. Maybe the difference in ratio of correct recombinants can be attributed to different gene expression regulation system used for controlling lysis gene $E$. After all, more lysis protein $E$ could be produced to kill 
bacteria under the control of strong promoter $p L$ compared with rigorous $P_{B A D}$ promoter.

Lysis gene $E$ could be used as a counter-selection marker both inE. coli and in $S$. marcescens. Since it has general lethal efficiency in Gram-negative strains, it is also applicable in other Gram-negative strains as long as promoter $p L$ and $P_{B A D}$ could work. Although a universal counter-selection gene based on $E$ was developed by us, there still existed escaping colonies during seamless modification, especially in $S$. marcescens. This leads to relative low ratio of correct recombinants during PCR identification when dsDNA mediated recombination was conducted in S. marcescens . Of course escaping colonies also existed in ssDNA mediated recombination, high ratio of correct recombinants is attributed to the high recombination efficiency of ssDNA itself (Ellis, Yu, \& DiTizio, 2001). It should be pointed out that in order to make the colonies exhibit clearly in the LB plate, only $5 \mu \mathrm{l}$ of recovered cells after transportation was plated in ssDNA recombination, while $100 \mu \mathrm{l}$ was plated in dsDNA recombination.

To provide a good alternative tool for seamless modification, counter-selection system based on lysis gene $E$ need to be optimized to improve its selection stringency. In our previous work, we developed a counterselection cassette based on kil gene of lambda phage. It performs well in $E$. coli and its selection stringency is comparable to the next best counter-selection systemtet $A-s a c B$. Both kil gene and $E$ gene are short in length: the CDS of kil and $E$ is $144 \mathrm{bp}$ and 273 bp separately. We believe that combining kil and $E$ gene in a certain way would be a good idea. An obvious benefit is that the selection stringency would be greatly improved. What is more, it will not bring any additional difficulties to the experimental process. After all, CDS length of the counter-selection cassette after combining the two genes is less than $500 \mathrm{bp}$, which is shorter than most of the counter-selection markers. We have tried to combine the two gene through fusion expression, however no selection stringency improvement was observed (data now shown). Maybe the function of proteins was affected by the changes in spatial conformation after fusion expression. We think co-express the two genes in the form of bi-cistron should be a better alternative solution from the perspective of reducing the background. Because nonsenses mutation in the first gene would not inactivate the second gene since independent products are translated, which is not applicable for fusion expression. Co-express kil and Egene through a classic RBS between their CDS indeed greatly improved the selection stringency to the level of the best reported toxins inducible system in E. coli $\left(4.9 \times 10^{-8}\right.$ at ack locus and $3.2 \times 10^{-8}$ at $\operatorname{ara} B$ locus). Surely, it is possible to obtain better selection stringency through combining $k i l$ and $E$ in other ways, such as improving translation initiation of the second gene using "bicistronic design" (BCD) reported by Mutalik (Mutalik et al., 2013). Many other strategies could also be used, such as combining more counter-selection markers with short size in length. Our work, in this paper, mainly aims to provide an example for improving selection stringency based on existing markers.

Other than developing the high selection-stringent kil-sd-Ecounter-selection cassette under the control of $p L$ promoter in this paper, we also constructed AraC/PBAD controlling kil-sd-Emarker which could be used in bacteria where high temperature is not suitable for growth. In S. marcescens, it does not perform as excellent as $p L$ promoted kil-sd-E . By introducingaraC gene harboring plasmid, the selection stringency was improved 4- to 170- fold. In particular, the selection stringency at these loci tested in this paper is all comparable to the best counter-selection system, inducible toxins system. At araB locus in E. coli and marR-1 locus in $S$. marcescens, it reaches the level of $10^{-9}$, the lowest selection stringency frequency for counter-selection so far.

This gives us an enlightenment: we could integrate the regulating elements of counter-selection markers, such as $\mathrm{araC}$ gene, into Red recombinases genes harboring plasmid. Therefore, counter-selection cassette will be reduced greatly in size. Take AraC/P $\mathrm{P}_{\mathrm{BAD}}-k i l-s d-E-G m^{R}$ double selection cassette as an example, if araC gene was integrated into the Red recombination system providing plasmid, only 1,327 bp $\mathrm{P}_{\mathrm{BAD}}$-kil$s d-E-G m^{R}$ cassette was needed for seamless modification. This will not only facilitate PCR amplification, but also improve recombination efficiency because longer substrate dsDNA decreases the chances of a $\lambda$ Red recombination event (Wei Chen et al., 2019).

\section{Conclusions}


In conclusion, we have developed a new counter-selection system based on lysis gene $E$. In view of the generic lethality to Gram-negative bacteria, it is very promising to be a universal alternative for genomic seamless modification. Counter-selection cassette based on lysis gene $E$ under two sets of expression regulation system, $\mathrm{cI} / \mathrm{pL}$ and Arac/ $\mathrm{P}_{\mathrm{BAD}}$, were developed to adapt to different hosts. Seamless modification was conducted successfully and the highest selection stringency so far was obtained both in E. coli and in S. marcescens .

\section{ACKNOWLEDGEMENTS}

We would like to thank Prof. Donald L. Court for providing plasmid pKD46, strains DY330 and W3110. Special thanks to Professor Sheng Yang for providing the strain MG1655.

\section{CONFLICT OF INTERESTS}

The authors declare that there are no conflict of interests.

\section{AUTHORS CONTRIBUTIONS}

Wei Chen, Ruyi Chen and Xiaotong Wu conducted the experiments; Wei Chen designed the experiments and wrote the manuscript. Ling He revised the manuscript. All authors read and approved the final manuscript.

\section{CONSENT FOR PUBLICATION}

All the co-authors approved to published this work inBiotechnology Journal .

\section{FUNDING}

This study was supported by the Science and Technology Program of Guangdong Province (2015A010107014), Grant-in-Aid from the Natural Scientific Foundation of China (31501895).

\section{References}

Blasi, U., Linke, R. P., \& Lubitz, W. (1989). Evidence for membrane-bound oligomerization of bacteriophage phi X174 lysis protein-E. Journal of Biological Chemistry, 264 (8), 4552-4558. Retrieved from https://www.ncbi.nlm.nih.gov/pubmed/2466836

Chen, W., Chen, R., \& Cao, J. (2021). Rapid Genome Modification in Serratia marcescens Through Red Homologous Recombination. Applied Biochemistry and Biotechnology . doi:10.1007/s12010-021-03576-y

Chen, W., Li, Y., Wu, G., Zhao, L., Lu, L., Wang, P., . . . Li, S. (2019). Simple and efficient genome recombineering using kil counter-selection in Escherichia coli.Journal of Biotechnology, 294, 58-66.

Chen, Z., Ling, W., \& Shang, G. (2016). Recombineering and I-SceI-mediated Pseudomonas putida KT2440 scarless gene deletion. FEMS Microbiology Letters, 363 (21). doi:10.1093/femsle/fnw231

DeVito, J. A. (2008). Recombineering with tolC as a selectable/counter-selectable marker: remodeling the rRNA operons of Escherichia coli. Nucleic Acids Research, 36 (1), e4-e4.

Ellis, H. M., Yu, D., \& DiTizio, T. (2001). High efficiency mutagenesis, repair, and engineering of chromosomal DNA using single-stranded oligonucleotides.Proceedings of the National Academy of Sciences, 98 (12), $6742-6746$.

Emruzi, Z., Aminzadeh, S., Karkhane, A. A., Alikhajeh, J., Haghbeen, K., \& Gholami, D. (2018). Improving the thermostability of Serratia marcescens B4A chitinase via G191V site-directed mutagenesis. International Journal of Biological Macromolecules, 116 , 64-70. doi:10.1016/j.ijbiomac.2018.05.014

Haeusser, D. P., Hoashi, M., Weaver, A., Brown, N., Pan, J., Sawitzke, J. A., . . . Margolin, W. (2014). The Kil peptide of bacteriophage $\lambda$ blocks Escherichia coli cytokinesis via ZipA-dependent inhibition of FtsZ assembly. PLoS Genetics, 10 (3), e1004217.

Hajam, I. A., Dar, P. A., Won, G., \& Lee, J. H. (2017). Bacterial ghosts as adjuvants: mechanisms and potential. Veterinary Research, 48 . doi:ARTN 37 


\section{$10.1186 / \mathrm{s} 13567-017-0442-5$}

Heap, J. T., Ehsaan, M., Cooksley, C. M., Ng, Y.-K., Cartman, S. T., Winzer, K., \& Minton, N. P. J. N. a. r. (2012). Integration of DNA into bacterial chromosomes from plasmids without a counter-selection marker. 40 (8), e59-e59.

Henrich, B., Lubitz, W., \& Plapp, R. (1982). Lysis of Escherichia coli by induction of cloned phi X174 genes. Molecular and General Genetics, 185 (3), 493-497. doi:10.1007/BF00334146

Hutchison, C. A., 3rd, \& Sinsheimer, R. L. (1966). The process of infection with bacteriophage phi-X174. X. Mutations in a phi-X Lysis gene. Journal of Molecular Biology, 18 (3), 429-447. doi:10.1016/s00222836(66)80035-9

Imam, A. A., Patrinos, G. P., de Krom, M., Bottardi, S., Janssens, R. J., Katsantoni, E., . . . Grosveld, F. G. (2000). Modification of human $\beta$-globin locus PAC clones by homologous recombination in Escherichia coli. Nucleic Acids Research, 28 (12), e65-e65.

Khetrapal, V., Mehershahi, K., Rafee, S., Chen, S., Lim, C. L., \& Chen, S. L. (2015). A set of powerful negative selection systems for unmodified Enterobacteriaceae.Nucleic Acids Research, 43 (13), e83-e83.

Langemann, T., Koller, V. J., Muhammad, A., Kudela, P., Mayr, U. B., \& Lubitz, W. (2010). The Bacterial Ghost platform system: production and applications.Bioeng Bugs, 1 (5), 326-336. doi:10.4161/bbug.1.5.12540

Lee, E.-C., Yu, D., De Velasco, J. M., Tessarollo, L., Swing, D. A., Court, D. L., . . . Copeland, N. G. (2001). A highly efficient Escherichia coli-based chromosome engineering system adapted for recombinogenic targeting and subcloning of BAC DNA.Genomics, 73 (1), 56-65.

Li, X.-t., Thomason, L. C., Sawitzke, J. A., \& Costantino, N. (2013). Positive and negative selection using the tetA-sacB cassette: recombineering and P1 transduction in Escherichia coli. Nucleic Acids Research, 41 (22), e204-e204.

Ma, W., Wang, X., Mao, Y., Wang, Z., Chen, T., \& Zhao, X. (2015). Development of a markerless gene replacement system in Corynebacterium glutamicum using upp as a counter-selection marker. Biotechnology Letters, 37 (3), 609-617. doi:10.1007/s10529-014-1718-8

Meyers, E. N., Lewandoski, M., \& Martin, G. R. (1998). An Fgf8 mutant allelic series generated by Creand Flp-mediated recombination. Nature Genetics, 18 (2), 136-141. doi:10.1038/ng0298-136

Mutalik, V. K., Guimaraes, J. C., Cambray, G., Lam, C., Christoffersen, M. J., Mai, Q. A., . . . Endy, D. (2013). Precise and reliable gene expression via standard transcription and translation initiation elements. Nature Methods, 10 (4), 354-360. doi:10.1038/nmeth.2404

Muyrers, J. P., Zhang, Y., Benes, V., Testa, G., Ansorge, W., \& Stewart, A. F. (2000). Point mutation of bacterial artificial chromosomes by ET recombination. EMBO reports, 1 (3), 239-243.

Pan, X., Sun, C., Tang, M., You, J., Osire, T., Zhao, Y., . . . Rao, Z. (2019). LysR-Type Transcriptional Regulator MetR Controls Prodigiosin Production, Methionine Biosynthesis, Cell Motility, H2O2 Tolerance, Heat Tolerance, and Exopolysaccharide Synthesis in Serratia marcescens. Applied and Environmental Microbiology, 86 (4). doi:10.1128/AEM.02241-19

Reisch, C. R., \& Prather, K. L. (2015). The no-SCAR (Scarless Cas9 Assisted Recombineering) system for genome editing in Escherichia coli. Scientific Reports, 5 , 15096. doi:10.1038/srep15096

Van Zyl, W. F., Dicks, L. M., \& Deane, S. M. J. B. m. b. (2019). Development of a novel selection/counterselection system for chromosomal gene integrations and deletions in lactic acid bacteria. 20 (1), 1-16.

Velez-Gomez, J. M., Melchor-Moncada, J. J., Veloza, L. A., \& Sepulveda-Arias, J. C. (2019). Corrigendum to "Purification and characterization of a metalloprotease produced by the C8 isolate of Serratia marcescens 
using silkworm pupae or casein as a protein source" [Int. J. Biol. Macromol. 135 (2019) 97-105].International Journal of Biological Macromolecules, 138 , 1142. doi:10.1016/j.ijbiomac.2019.07.142

Wang, H., Bian, X., Xia, L., Ding, X., Müller, R., Zhang, Y., . . Stewart, A. F. (2014). Improved seamless mutagenesis by recombineering using ccdB for counterselection.Nucleic Acids Research, 42 (5), e37-e37.

Warming, S., Costantino, N., Court, D. L., Jenkins, N. A., \& Copeland, N. G. (2005). Simple and highly efficient BAC recombineering using galK selection. Nucleic Acids Research, 33 (4), e36. doi:10.1093/nar/gni035

Witte, A., \& Lubitz, W. (1989). Biochemical characterization of phi X174-protein-E-mediated lysis of Escherichia coli. European Journal of Biochemistry, 180 (2), 393-398. doi:10.1111/j.1432-1033.1989.tb14661.x

Witte, A., Wanner, G., Sulzner, M., \& Lubitz, W. (1992). Dynamics of PhiX174 protein E-mediated lysis of Escherichia coli. Archives of Microbiology, 157 (4), 381-388. doi:10.1007/BF00248685

Won, G., Hajam, I. A., \& Lee, J. H. (2017). Improved lysis efficiency and immunogenicity of Salmonella ghosts mediated by co-expression of lambda phage holin-endolysin and X174 gene E. Scientific Reports, 7 , 45139. doi:10.1038/srep45139

Wong, Q. N., Ng, V. C., Lin, M. C., Kung, H.-f., Chan, D., \& Huang, J.-D. (2005). Efficient and seamless DNA recombineering using a thymidylate synthase A selection system in Escherichia coli. Nucleic Acids Research, 33 (6), e59-e59.

Yip, C.-H., Yarkoni, O., Ajioka, J., Wan, K.-L., Nathan, S. J. A. m., \& biotechnology. (2019). Recent advancements in high-level synthesis of the promising clinical drug, prodigiosin. 103 (4), 1667-1680.

Young, K. D., \& Young, R. (1982). Lytic action of cloned phi X174 gene E. Journal of Virology, 44 (3), 993-1002. doi:10.1128/JVI.44.3.993-1002.1982

Yu, D., Ellis, H. M., Lee, E. C., Jenkins, N. A., Copeland, N. G., \& Court, D. L. (2000). An efficient recombination system for chromosome engineering in Escherichia coli.Proceedings of the National Academy of Sciences of the United States of America, 97 (11), 5978-5983. doi:10.1073/pnas.100127597

Zhang, Y., Buchholz, F., Muyrers, J. P., \& Stewart, A. F. (1998). A new logic for DNA engineering using recombination in Escherichia coli. Nature Genetics, 20 (2), 123-128.

Zhang, Y., Buchholz, F., Muyrers, J. P., \& Stewart, A. F. J. N. g. (1998). A new logic for DNA engineering using recombination in Escherichia coli. 20 (2), 123-128.

Tables

Table 1 Bacterial strains

Strain

MG-10 MG-3 MG-4A MG-4B MG-5 MG-6 MG-7 CWE-1 CWE-2 CWE-3 CWE-4 CW-1 GY1 GY4 GY4-1 GY5 GY5A GY

a: 1,000bp DNA fragments of catenin gene from Helicoverpa. armigera . b, 1,000bp DNA fragments of T7 RNA polymerase gene from E. coli strain BL21 (DE3).

Table 2. Oligonucleotides

Name

Sph-E Nco-E Ack-Gen-F Ack-E-R Ack-F Gen-F Ack-catF Ack-catR TetA-F Ack-R EG-pkd-E EG-pkd-G Pkd-cx Gen-R Pi

${ }^{a}$ Underlined sequences indicate the primers for PCR amplification. The bold sequences represent homologous regions to target locus. The "//" indicates the site for double selection cassette insertion. 


\section{Figure Legends}

Figure 1 Construction and identification of the tet-pL-Eselection/counter-selection cassette. (A) Schematic of constructing plasmid pBBR1-E. (B) Schematic of constructing the tet- $p L$-Eselection/counter-selection cassette. DNA fragment in the dotted box indicates the goal region of the constructed tet- $p L-E$ cassette. (C) PCR identification of strain CWE-1. The PCR products were analyzed on a $1 \%$ agarose gel.

Lane 1 shows a 851-bp amplicon in CWE-1, while no band is observed in the control strain MG-10 (Lane Con). M represents the DNA marker. (D) PCR identification of strain CWE-2. The PCR products were analyzed on a $1 \%$ agarose gel. Lane 1 shows a 1,430-bp amplicon in CWE-2, while no band is observed in the control strain MG1655 (Lane Con). M represents the DNA marker.

Figure 2 Potential and functional testing of lysis gene $E$ as a counter-selection marker gene. (A) Cells were grown overnight at $30{ }^{\circ} \mathrm{C}$ or $42{ }^{\circ} \mathrm{C}$ after streak cultivation. (B) Serial spot dilution of cells was grown in LB agar plates at $30{ }^{\circ} \mathrm{C}$ or $42{ }^{\circ} \mathrm{C}$. (C) Colony formation of CWE-2 at $42{ }^{\circ} \mathrm{C}$ after Red recombination. No DNA (left) or 1,000 bp DNA fragment of catenin gene from $H$. armigera with 38-bp homologous to ack (right) was electroporated into competent CWE- 2 cells. $100 \mu \mathrm{L}$ of recovered cells were then plated onto agar plates and cultivated at $42{ }^{\circ} \mathrm{C}$. (D) PCR identification of the genomic substitution mentioned in (C). A total of 10 colonies (lane 1 to lane 10) were screened and a 1,116-bp product indicates successful substitution. Lane Con shows a 2,270-bp product in CWE-2. M represents the DNA marker.

Figure 3 Construction and functional testing of the $P_{B A D}-E-G m^{R}$ selection/counter-selection cassette. (A) Schematic of constructing plasmid pKD-EG. DNA fragment in the dotted box indicates the goal region of the constructed $P_{B A D}-E-G m^{R}$ cassette. (B) PCR identification of plasmid pKD-EG. The PCR products were analyzed on a $1 \%$ agarose gel. Lane 1 shows a 1,344-bp amplicon in pKD-EG, while no band is observed in the control plasmid pKD46 (Lane Con). M represents the DNA marker. (C) Induction of $E$ in plasmid pKD-EG with arabinose is lethal. GY1 [pKD-EG] was cultured overnight in LB broth without (left) or with the addition of $0.4 \%$ arabinose (right). (D) PCR identification of strain GY4. The PCR products were analyzed on a $1 \%$ agarose gel. Lane 1 shows a 1,610-bp amplicon in GY4, while no band is observed in the control strain GY1 (Lane Con). M represents the DNA marker. (E) Induction of $E$ in GY4 with arabinose is also lethal. GY14 was cultured overnight in LB broth without (left) or with the addition of $0.4 \%$ arabinose (right). (F) Serial spot dilution of GY4 and GY1 [pSim6] were grown in LB agar plates supplemented without (left) or with (right) the addition of $0.4 \%$ arabinose.

Figure 4 Genomic s eamless modification in S. marcescens chromosome. (A) Colony formation of GY4 after ssDNA-mediated recombination. $5 \mu \mathrm{L}$ of recovered cells were cultivated on LB agar plate supplemented with $0.4 \%$ arabinose. ssDNA represents GY4 transformed with oligo sspigA-F. Con represents GY4 transformed without DNA. (B) PCR detection of the $P_{B A D}-E-G m^{R}$ deletion using ssDNA. Lane Con shows a 3,477-bp amplicon in GY4, and a 1,190-bp product indicates successful deletion in Lane 1 to 10. M represents the DNA marker. (C) PCR detection of the $P_{B A D}-E-G m^{R}$ substitution by partial fragment of T7 RNA polymerase gene. Lane Con shows a 1,929-bp amplicon indicates successful substitution in Lane to 10, while no band is observed in the control strain GY5 (Lane Con). M represents the DNA marker.

Figure 5 Improving selection stringency frequency through combining $E$ and kil . Selection stringency frequency of $E$, kil and $k i l-s d-E$ was analyzed at ack (A), $a r a B(B)$ and pigA loci (D). Each point represents the mean \pm SD of three independent replicates. (C) MG-4A (left) and MG-4B (right) were cultured overnight in LB broth without or with the addition of $0.4 \%$ arabinose. (E) Colony formation of GY5 after ssDNAmediated recombination. $5 \mu \mathrm{L}$ of recovered cells were cultivated on LB agar plate supplemented with $0.4 \%$ arabinose. ssDNA represents GY5 transformed with oligo sspigA-F. Con represents GY5 transformed without DNA. (F) PCR detection of the $P_{B A D}-k i l-s d-E-G m^{R}$ deletion using ssDNA. Lane Con shows a 3,713-bp amplicon in GY5, and a 1,190-bp product indicates successful deletion in Lane 1 to 10 . M represents the DNA marker. (G) PCR detection of the $P_{B A D}-k i l-s d-E-G m^{R}$ substitution by partial fragment of T7 RNA polymerase gene. a 1,929-bp amplicon indicates successful substitution in Lane to 10, while no band is observed in the control strain GY5 (Lane Con). M represents the DNA marker. 
Figure 6 Improving selection stringency frequency of $k i l-s d-E$ by introducing plasmid pKDsg-ack. (A) Selection stringency frequency of kil-sd-E at $\operatorname{mar} R-1, r c s A$ and $h y p-1$ loci was analyzed. Each point represents the mean \pm SD of three independent replicates. (B) Selection stringency frequency of kil-sd- $E$ at araB locus in MG1655 [pSim6] and MG1655 [pKDsg-ack] was analyzed. Each point represents the mean \pm SD of three independent replicates. (C) Selection stringency frequency ofkil-sd-E at $\operatorname{marR-1}, r c s A$ and hyp-1 loci was analyzed. Each point represents the mean \pm SD of three independent replicates. (D) Comparation of selection stringency in $S$. marcescens carrying plasmid pSim6 or pKDsg-ack. The ratio is determined by dividing the selection stringency of the former by that of the latter.

Figure S1 Schematic of seamless deleting of $P_{B A D}-E-G m^{R}$ cassette by sspigA-F. $P_{B A D}-E-G m^{R}$ was inserted into the CDS of pigA (between the purple and green boxes). The "//" in sspig-F indicates the junction between the two homologies.

Figure S2 Construction of CWE-3. Schematic of constructing strain CWE-3 is shown in the left. RBS sequence (GAAGGAGATATACC) from pET32a was linked between the CDS of $E$ and $k i l$. DNA fragment in the dotted box indicates the goal region of the constructedkil-sd-E counter-selection cassette. PCR identification of the strain CWE-3 is shown in the right. Lane 1 shows an 851-bp amplicon in CWE-1, while no band is observed in the control strain MG-10 (Lane Con). M represents the DNA marker.

Figure S3 PCR identification of the strain MG-4A and MG-4B. Lane 1 shows an 360-bp (left) or an 600-bp (right) amplicon in MG-4A and MG-4B respectively, while no band is observed in the control strain MG1655 (Lane Con). M represents the DNA marker.

Figure S4 Colony formation of MG655 [pKDsg-ack]. Cells equivalent in quantity to $10^{-4} \mu \mathrm{L}$ of Overnightcultured MG655 [pKDsg-ack] were mixed with $100 \mu \mathrm{L}$ of fresh LB and then spread onto agar plate without or with the addition of $0.4 \%$ arabinose.

Fig. 1

A.

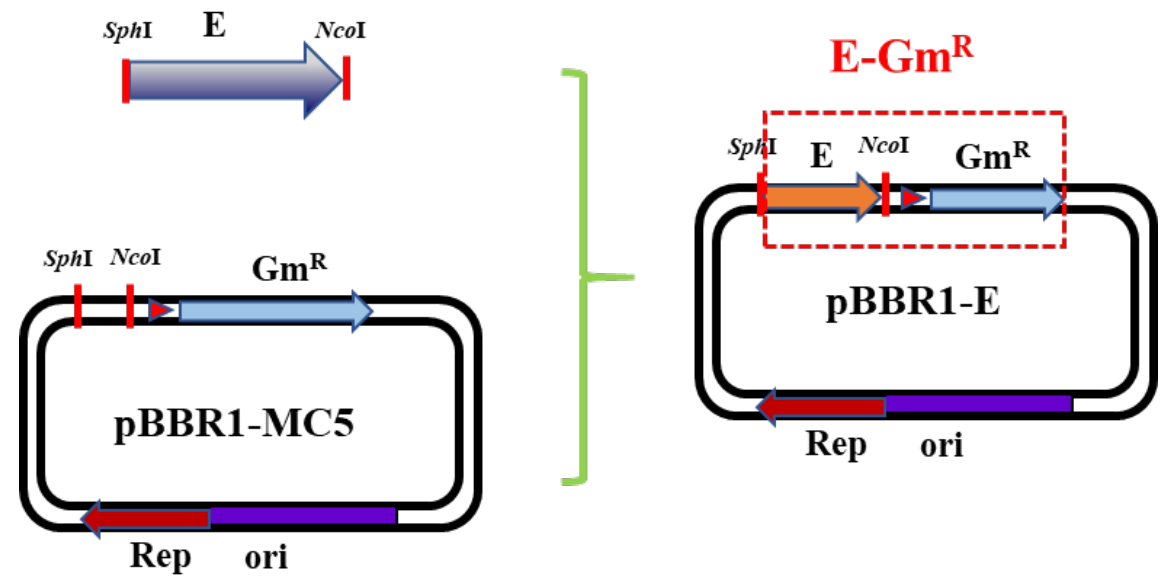


MG-10

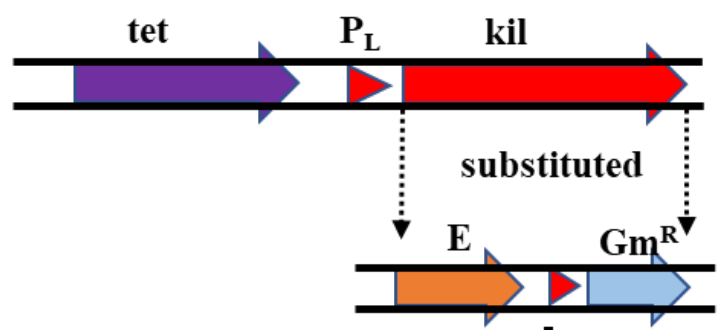

CWE-1

CWE-2

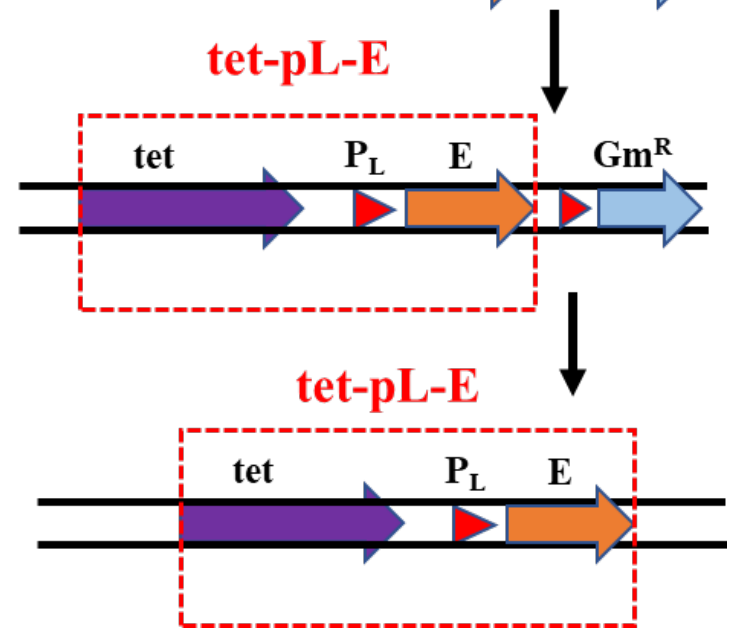

B.

C. D.

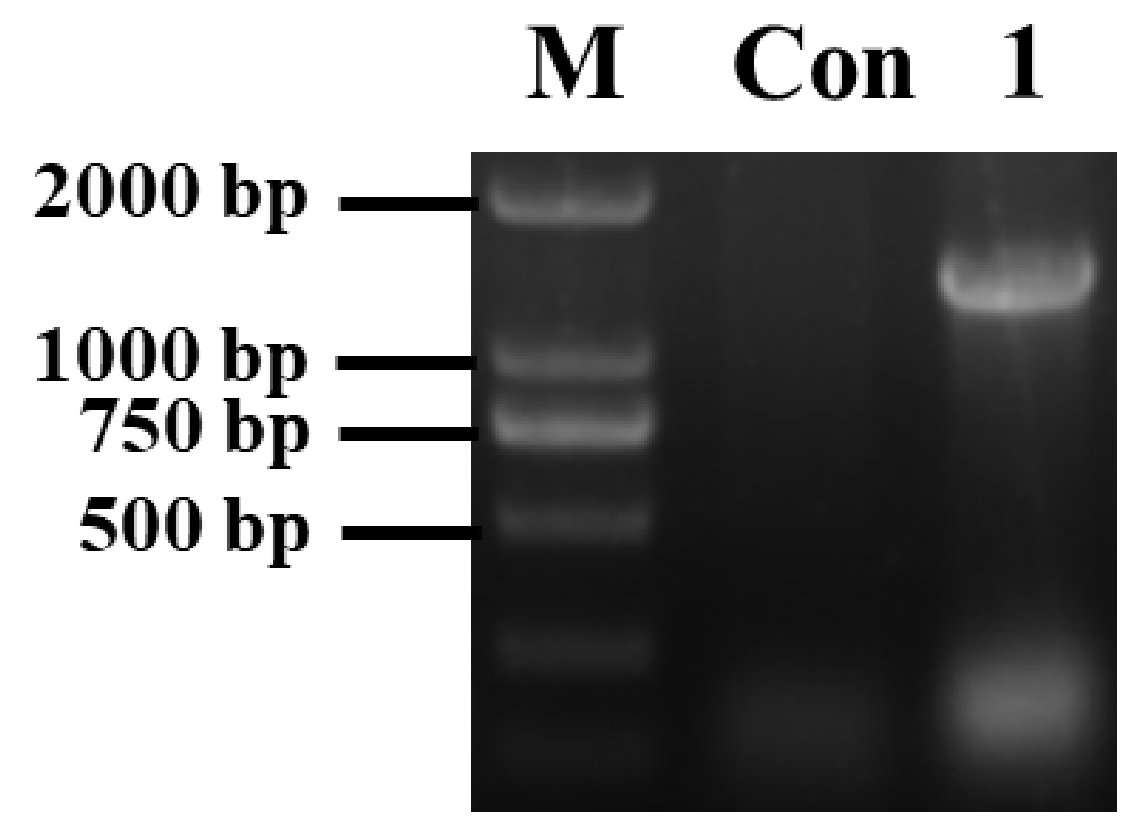




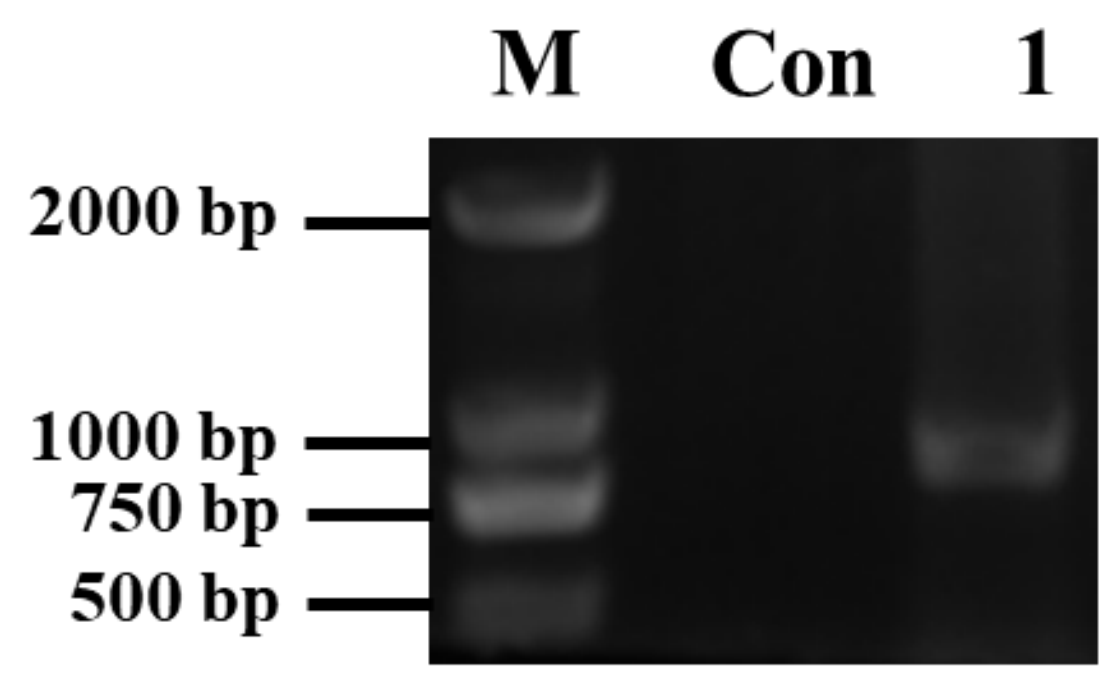

Fig. 2

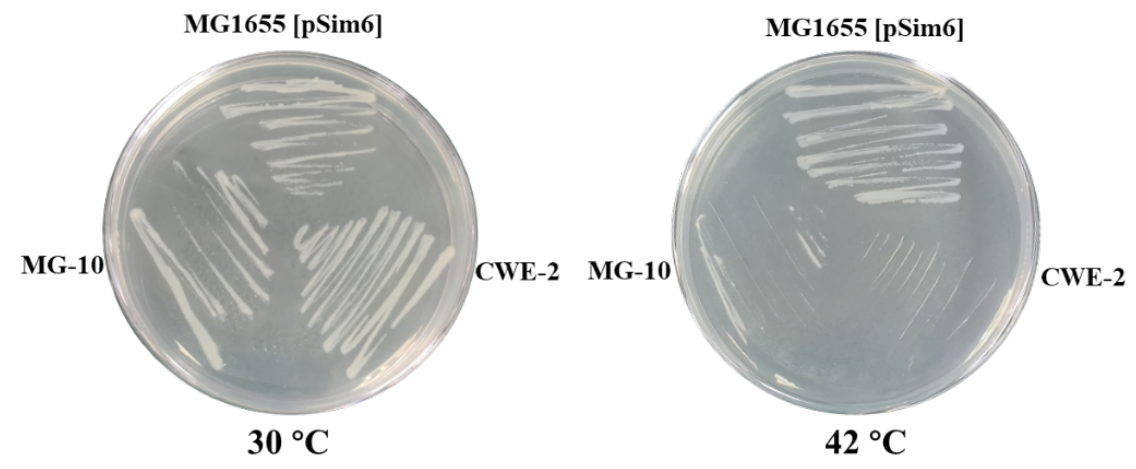

A.

B.
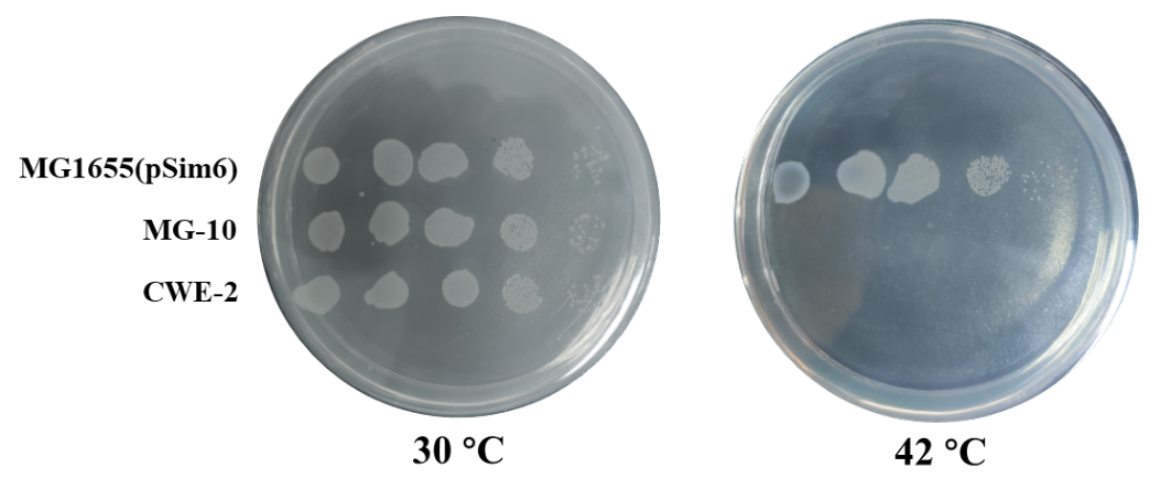

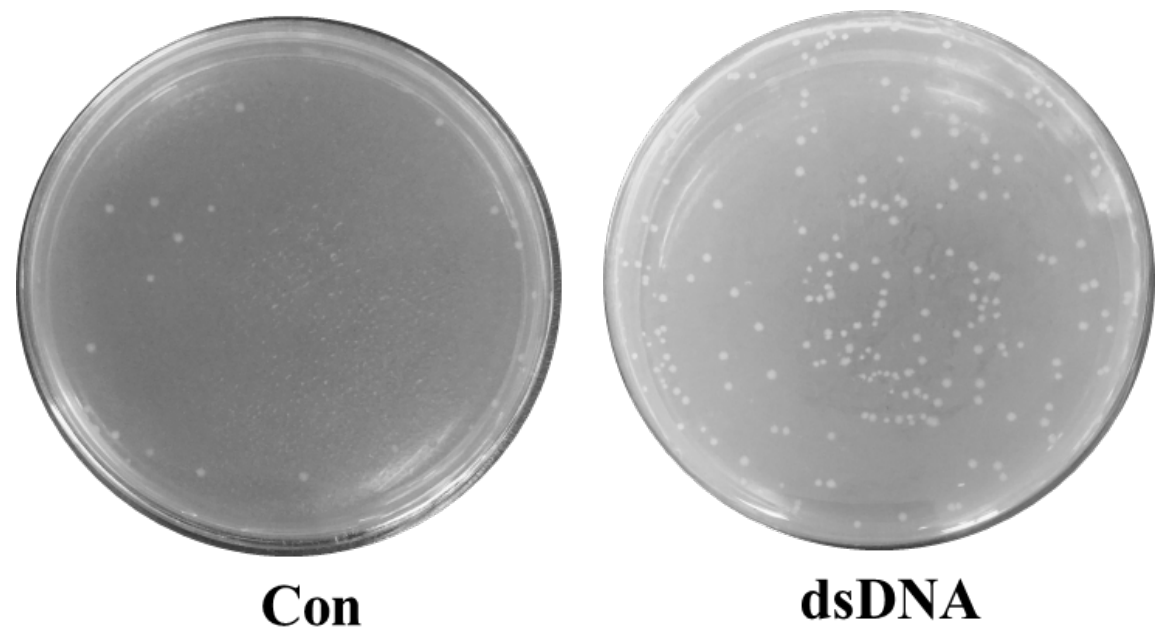

C.

D.

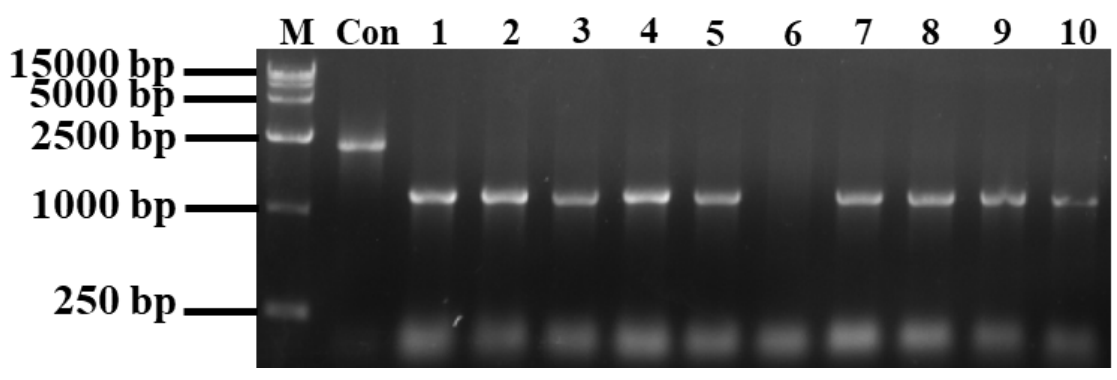

Fig. 3

A.
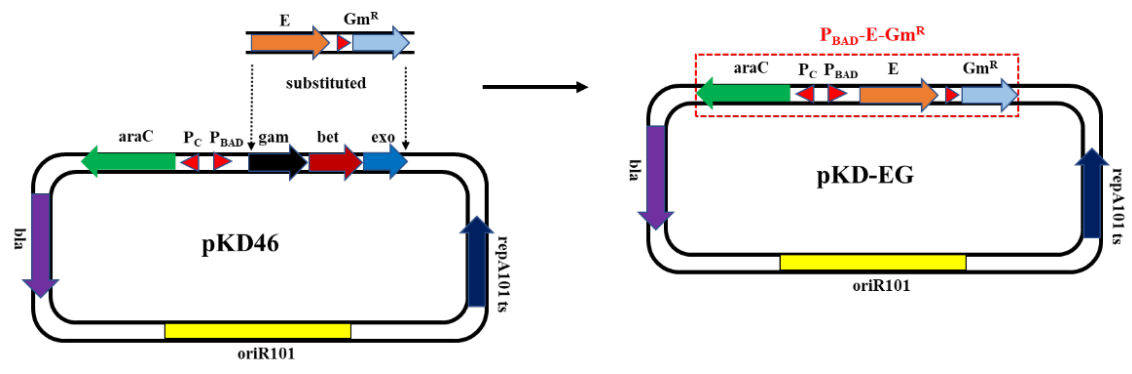


\section{arabinose}
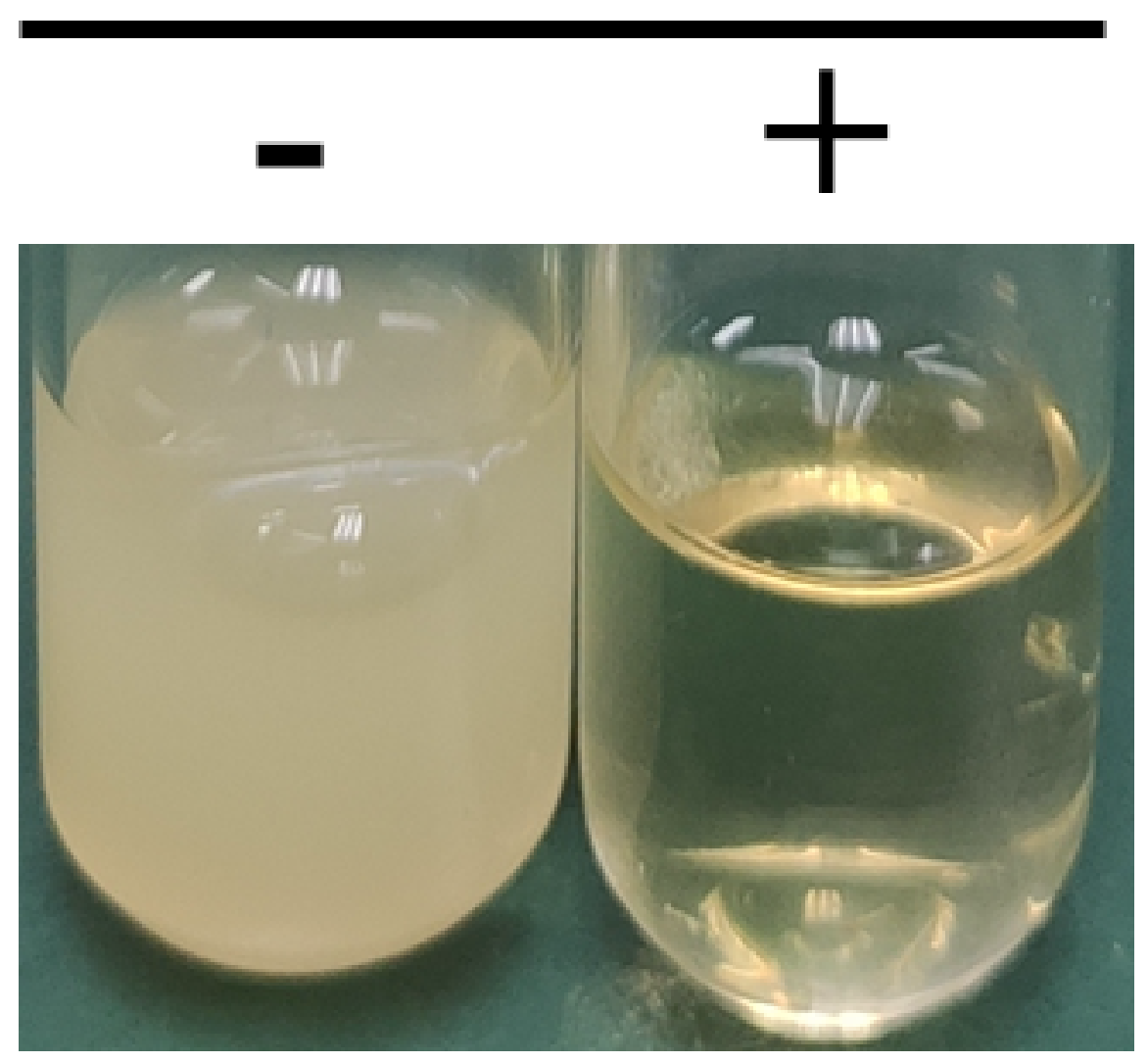

B.

C. 


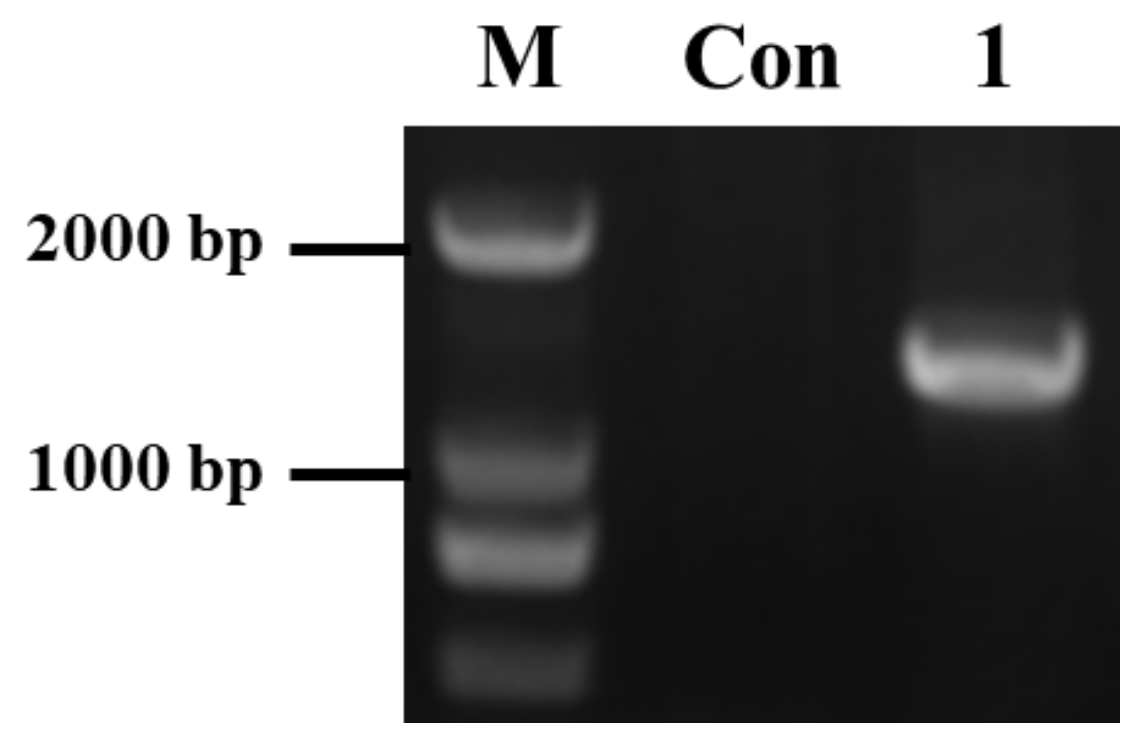

\section{arabinose}

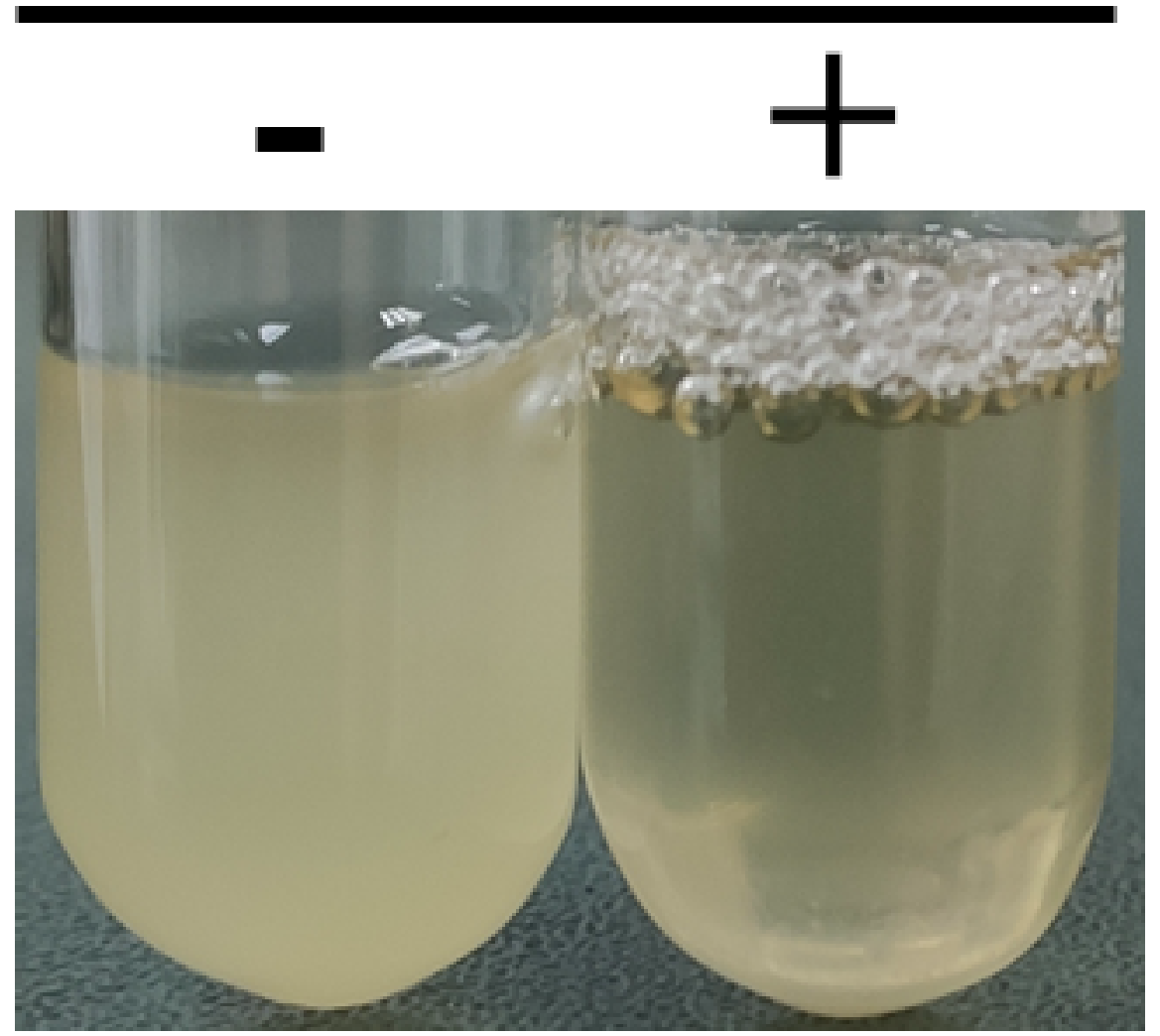




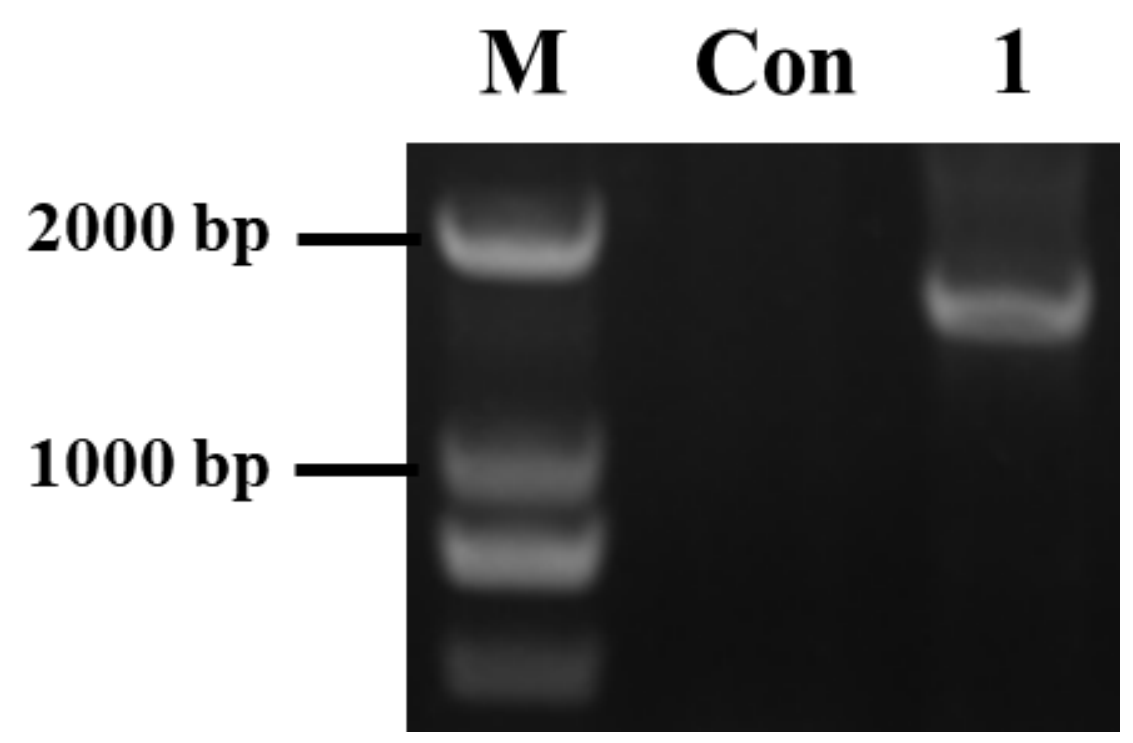

D.

E.

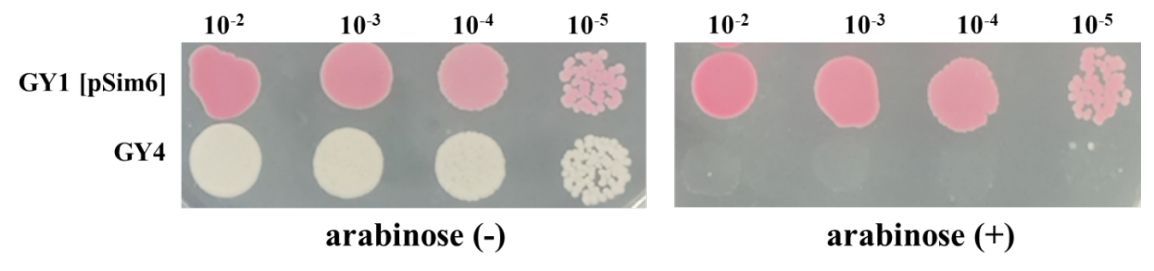

F.

Fig. 4

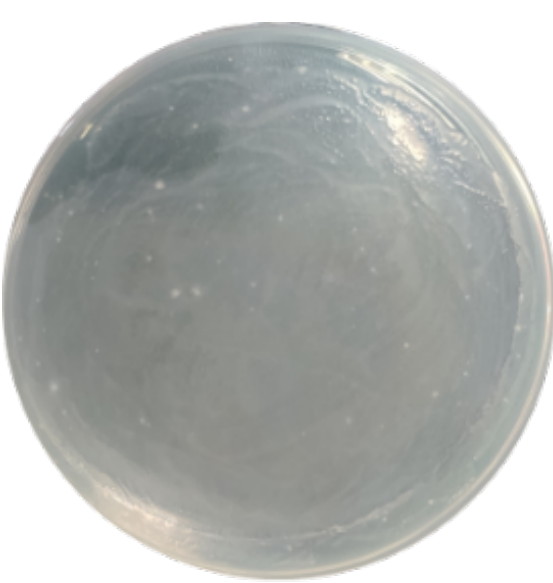

Con

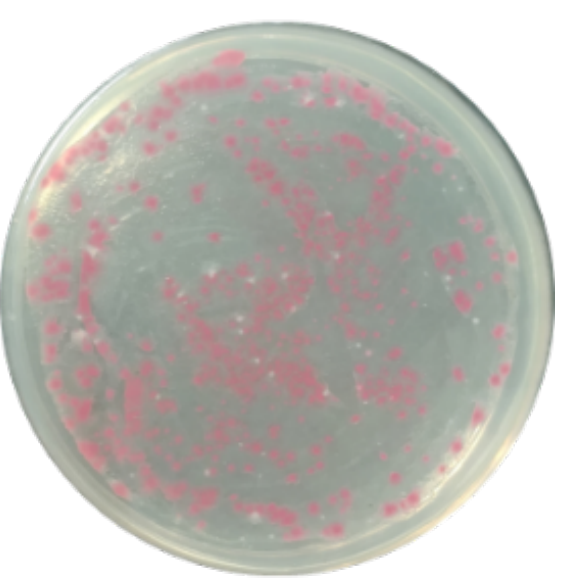

ssDNA 
A.

B.

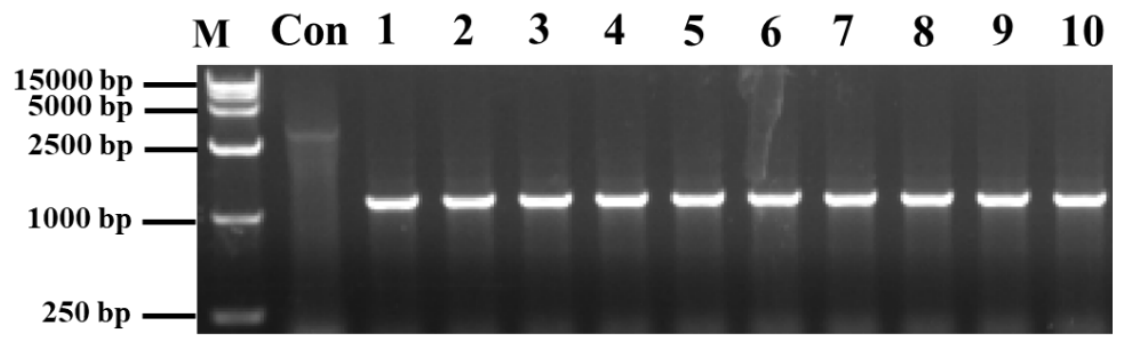

C.

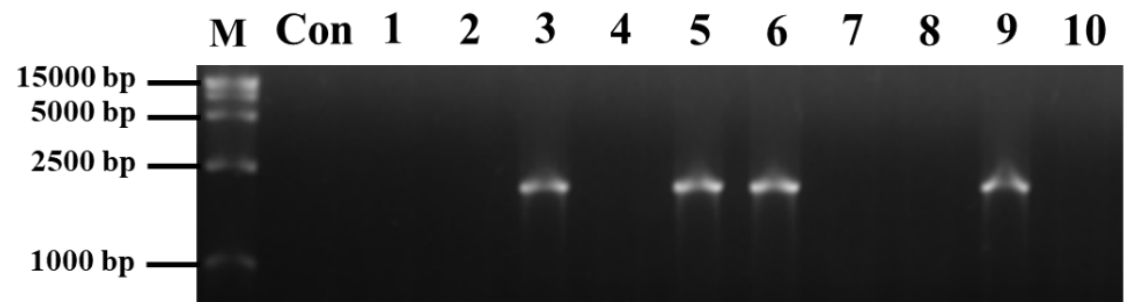

Fig. 5

A. B.

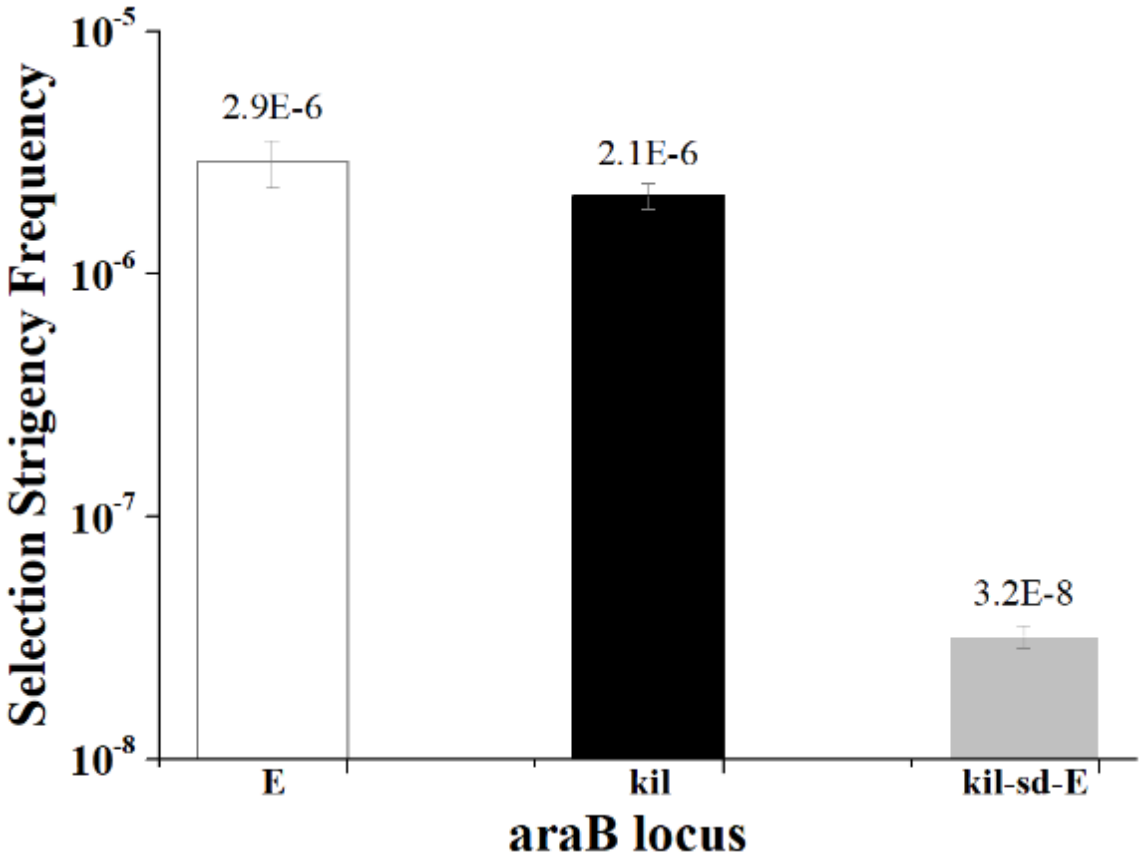




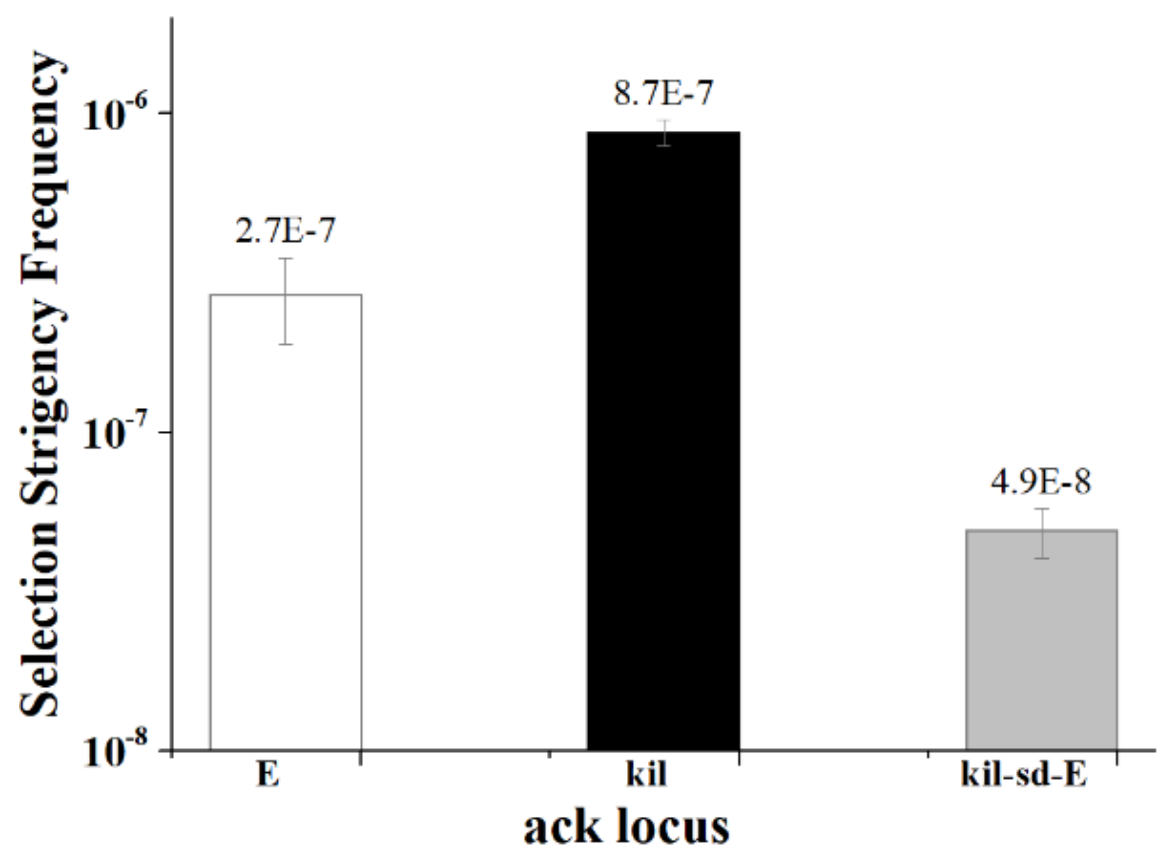

C. D.

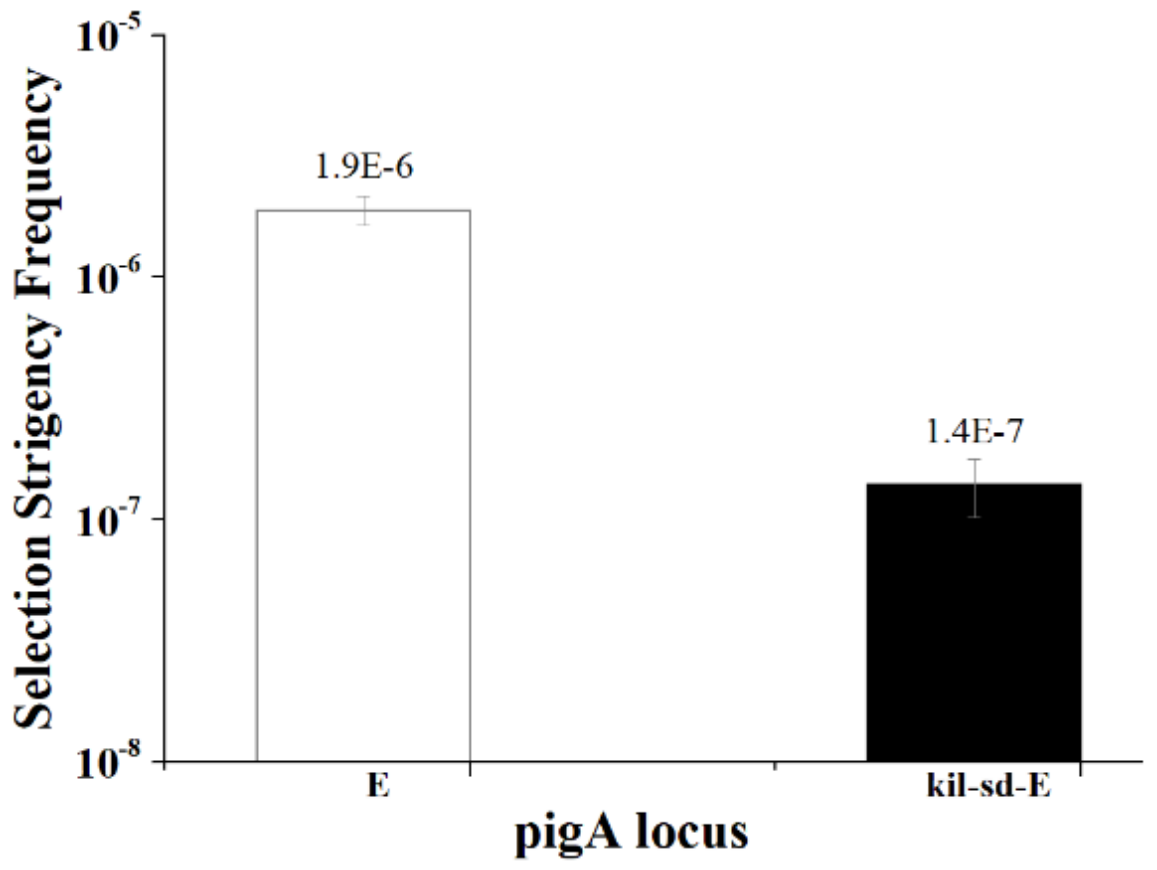




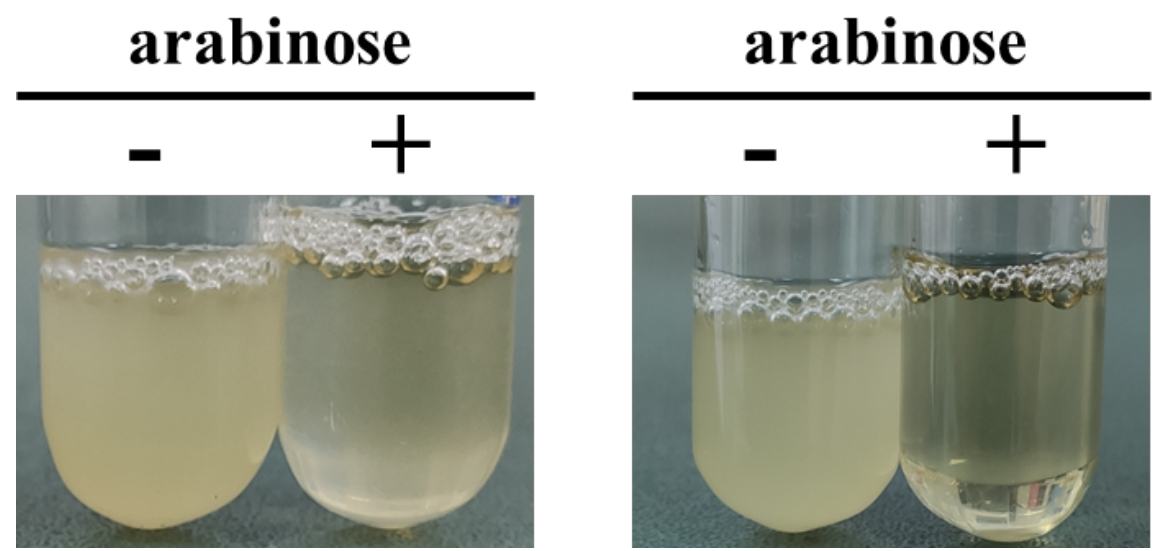

E.
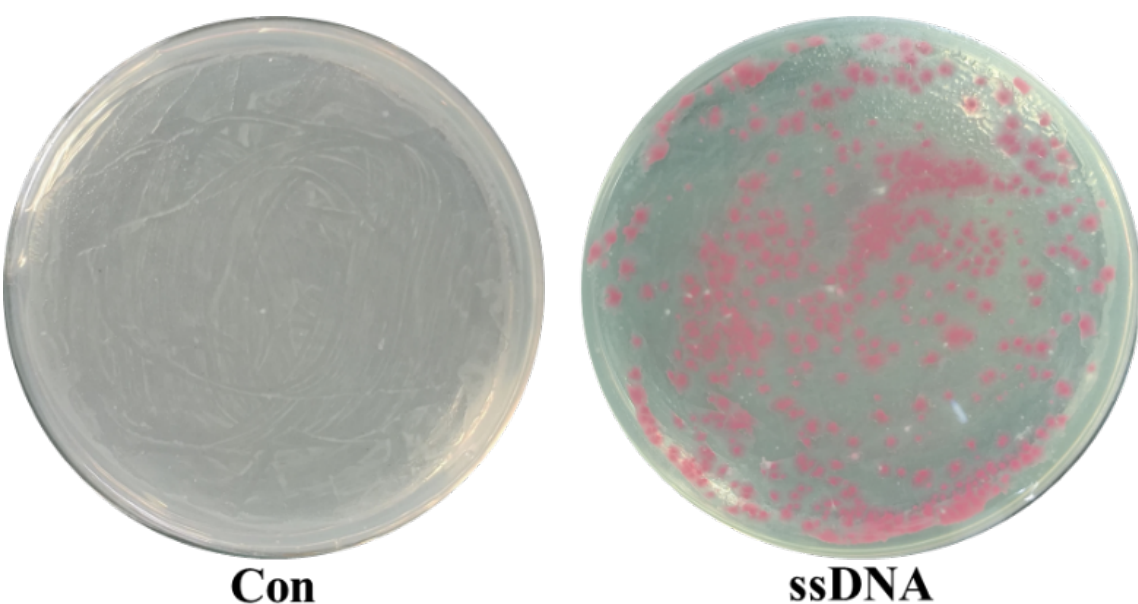

F.

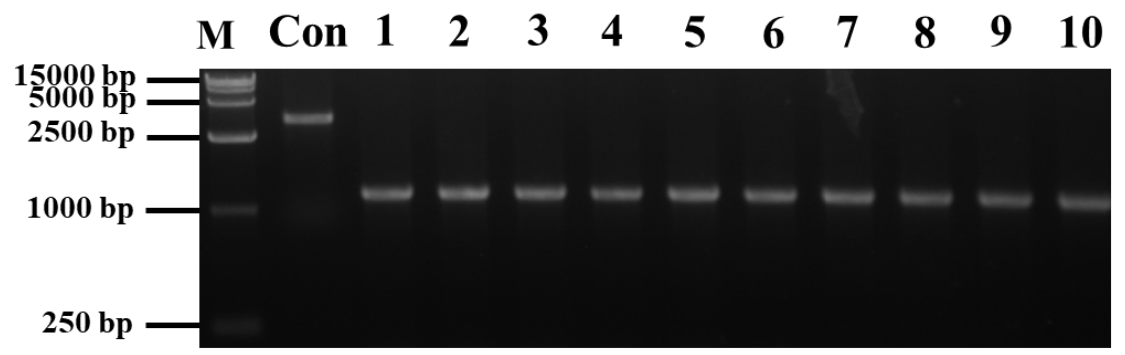




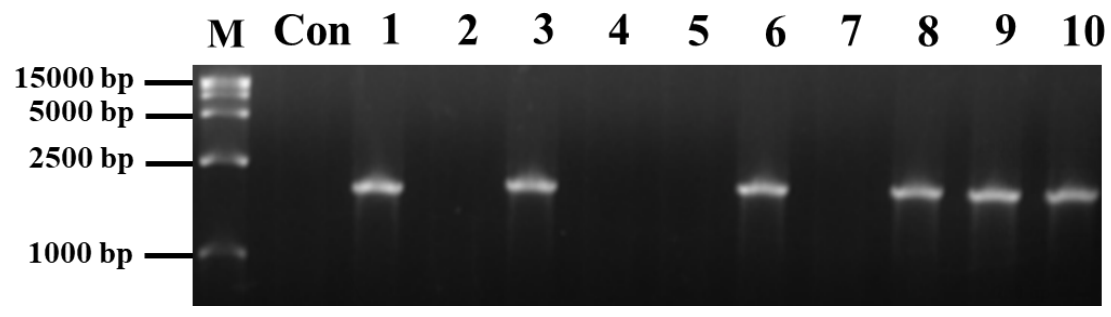

G.

Fig. 6

A. B.

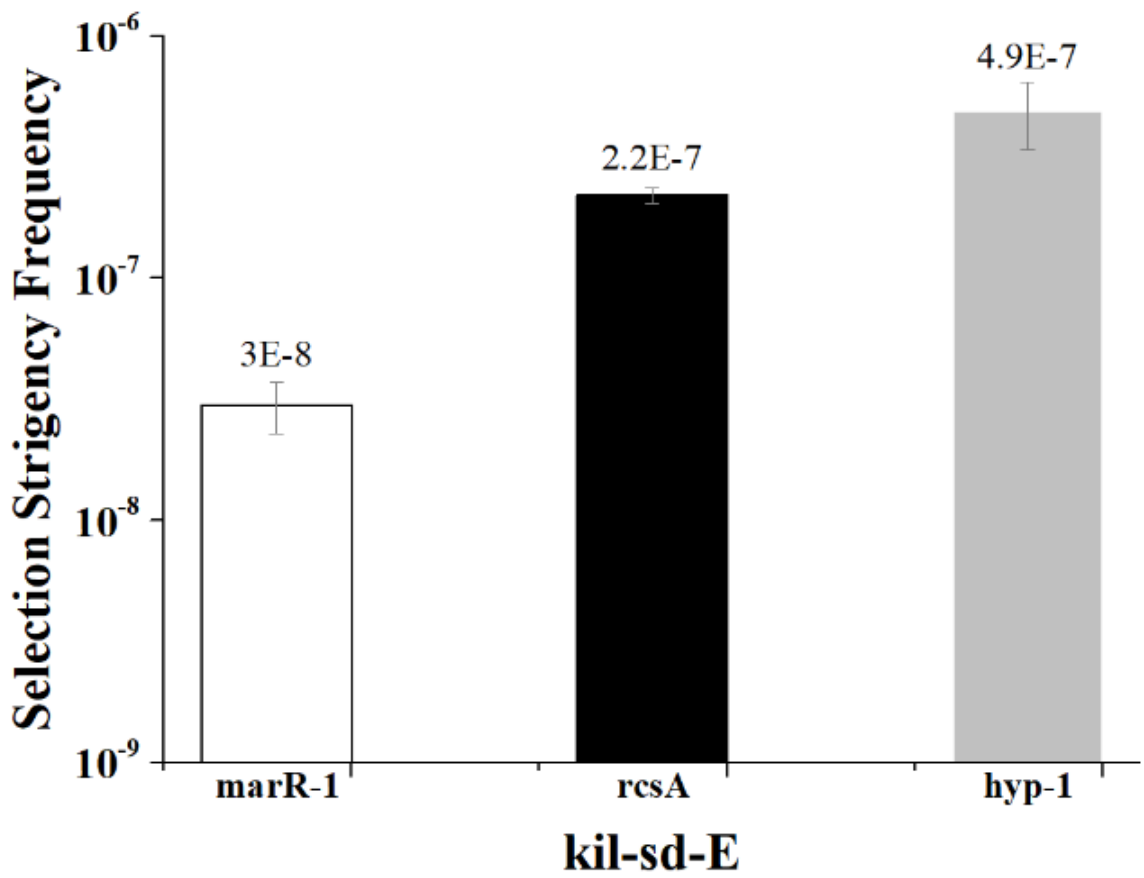




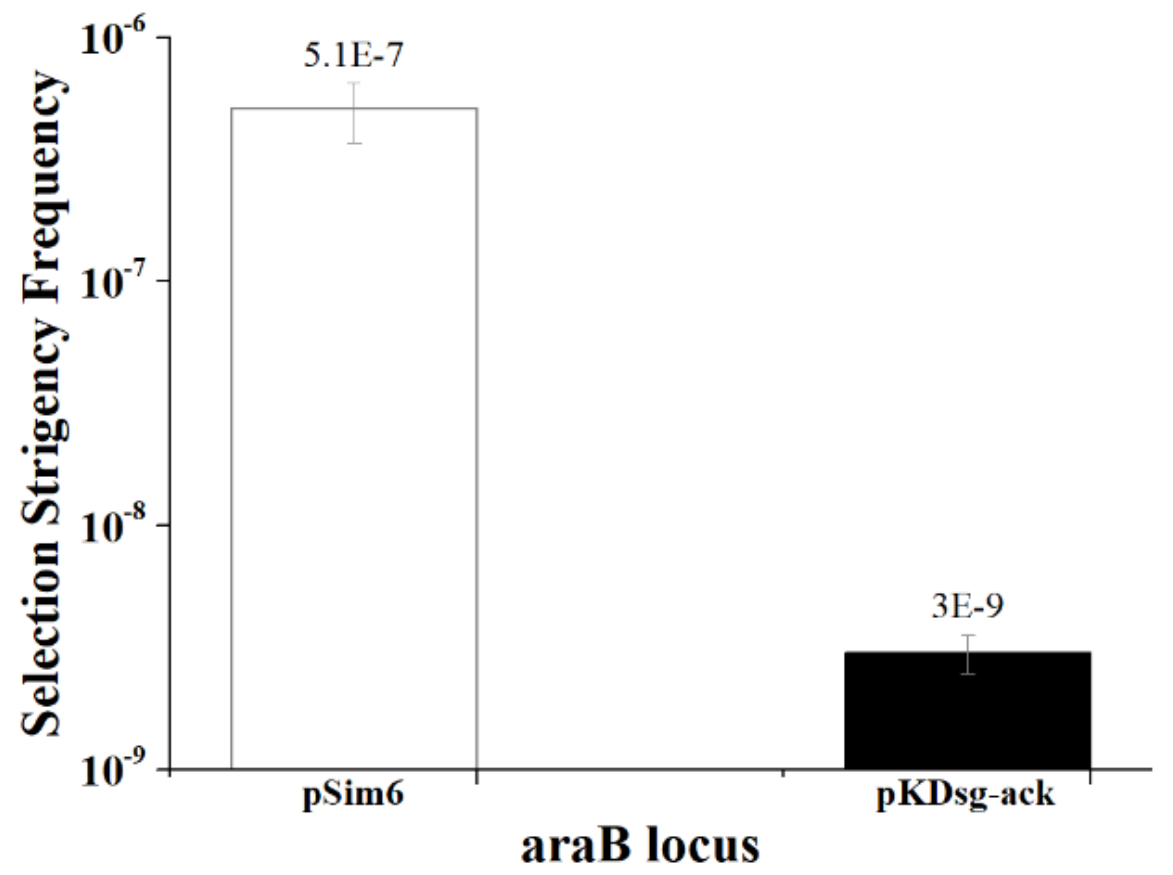

C. D.

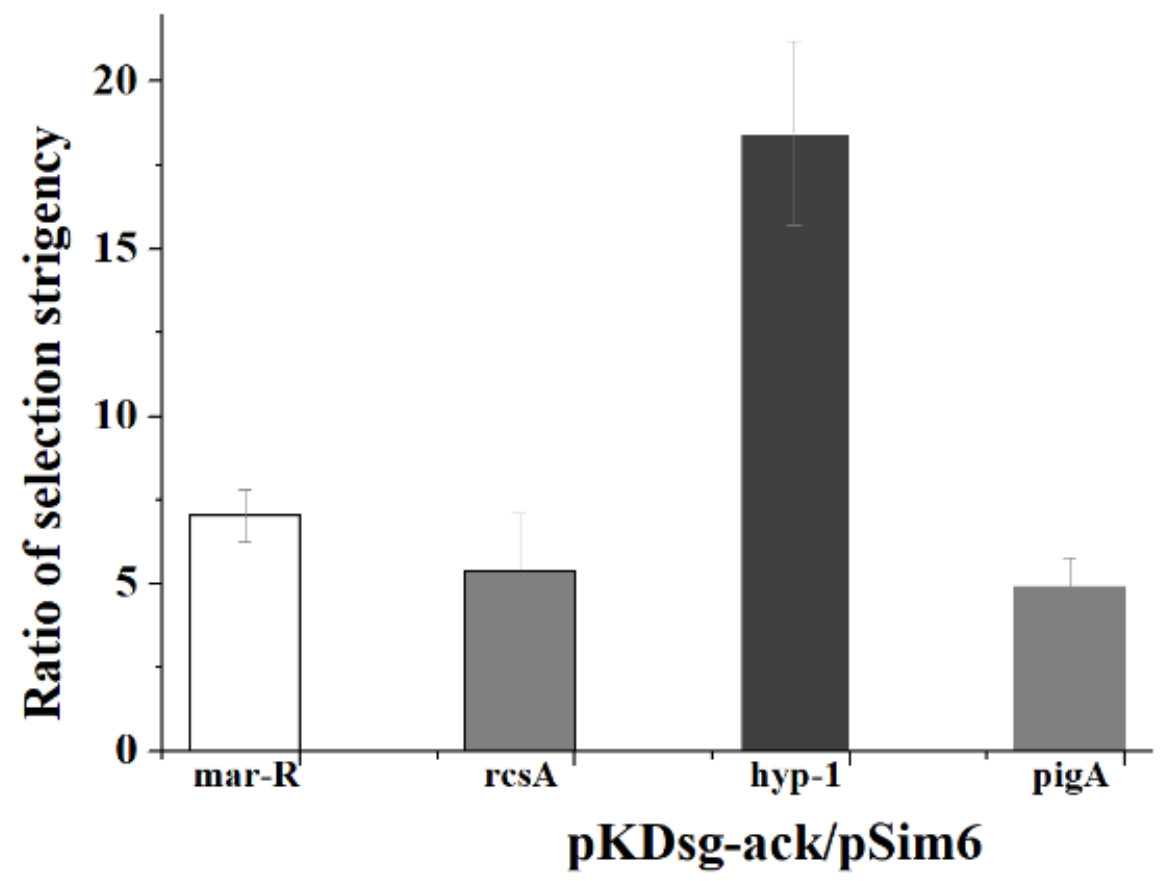




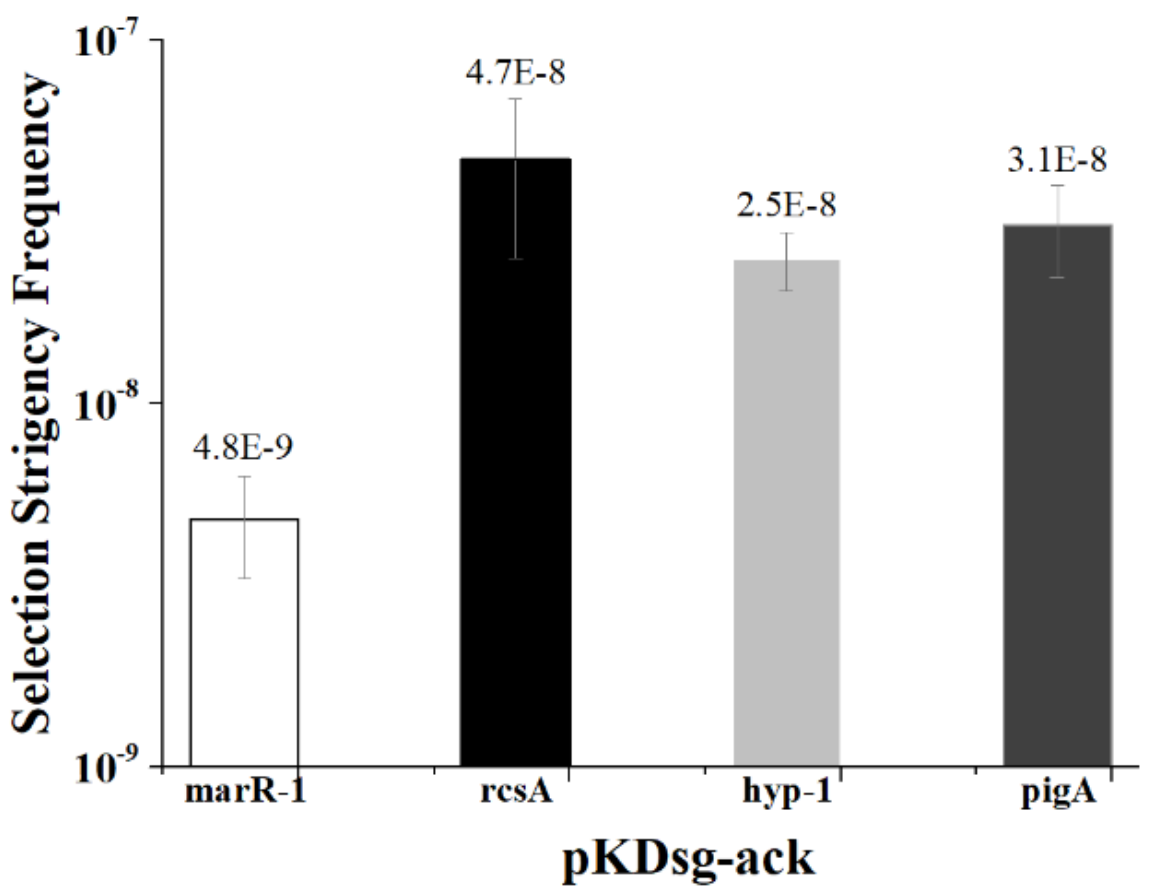

Fig. S1

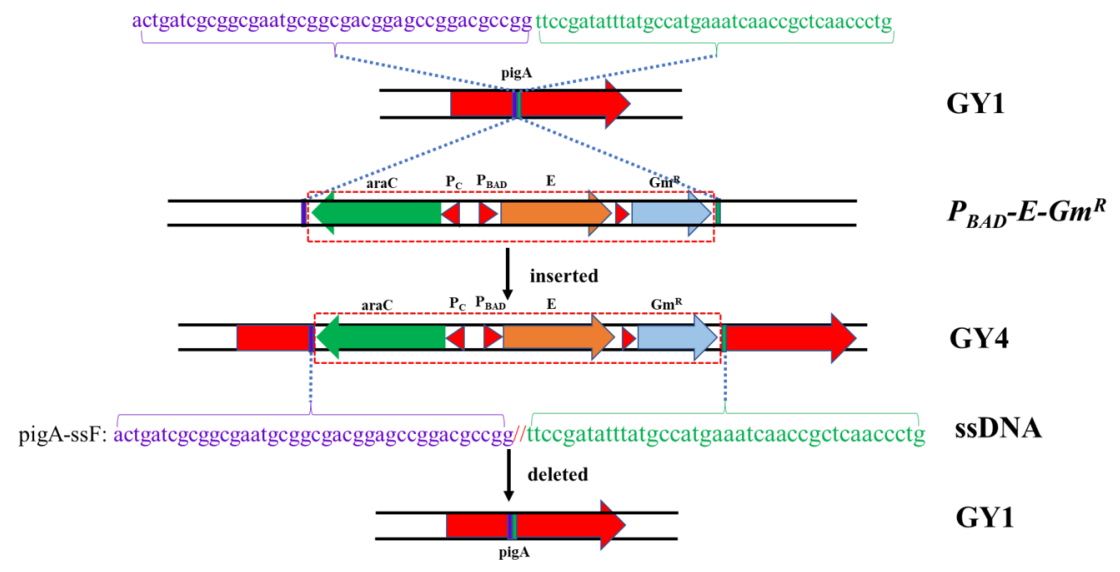

Fig. S2 

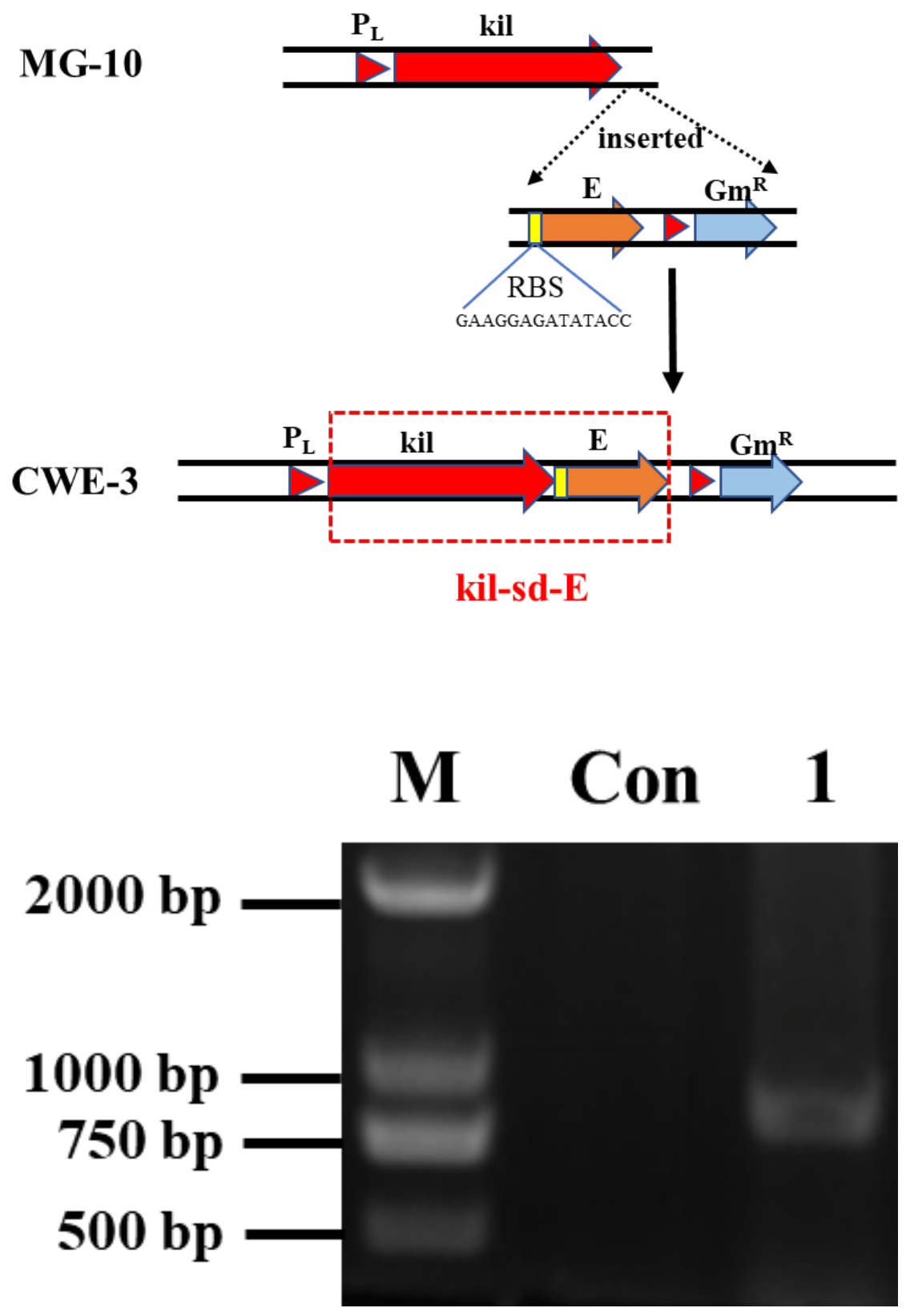

Fig. S3 


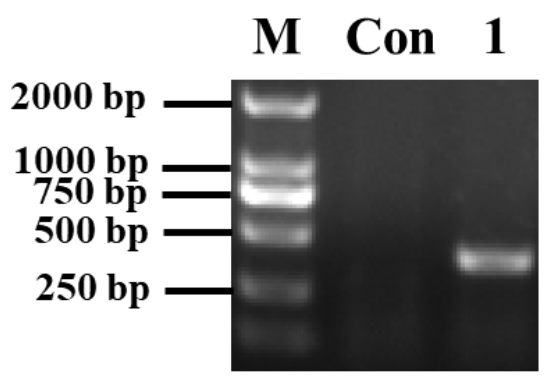

MG-4A

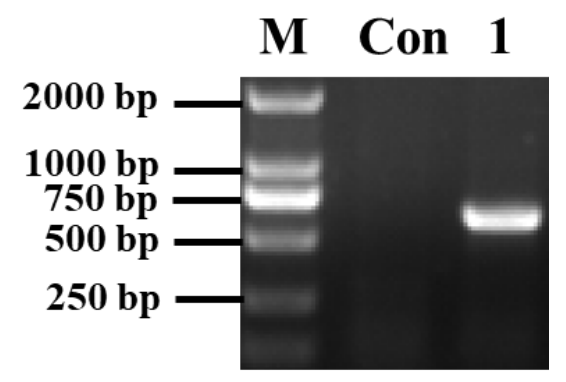

MG-4B

Fig. S4
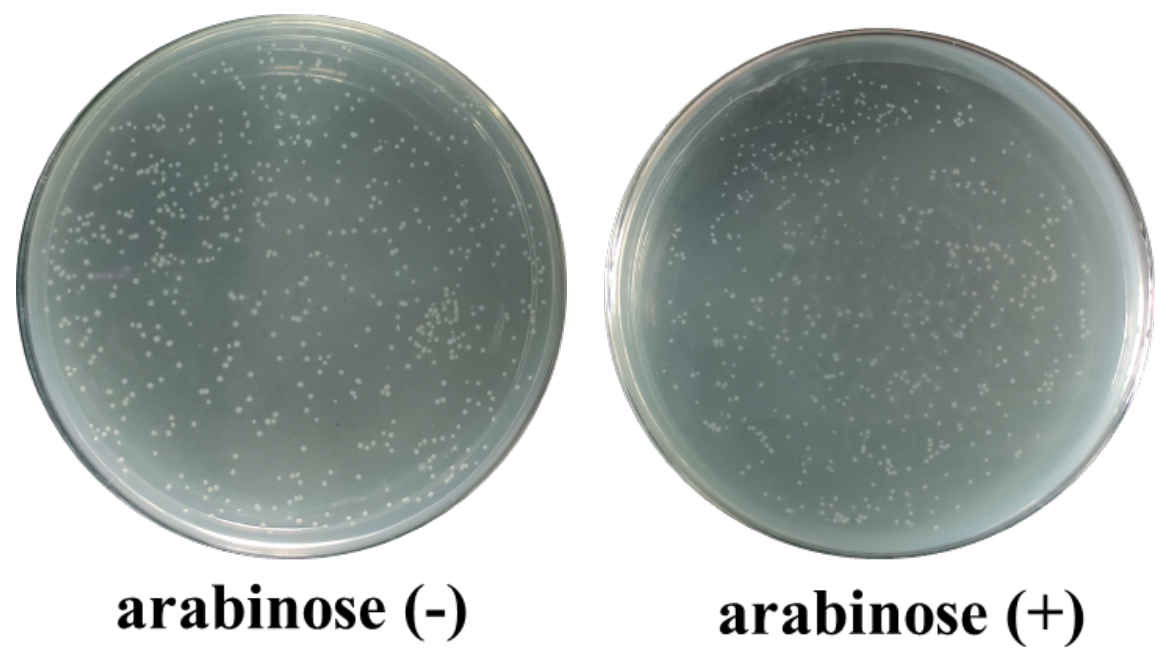